\title{
Ueber die Einwirkung verschiedener chemischer Stoffe auf die Thätigkeit des Mundspeichels.
}

Von

cand. med. F. Kübel aus Tübingen.

(Mit 8 Textfiguren.)

Wie bekannt, ist bei vielen Geschöpfen, zu denen auch der Mensch zählt, der Mundspeichel mit einer Eigenschaft ausgestattet, die man als diastatische bezeichnet. Diese diastatische Eigenschaft besteht darin, dass Stärkekleister ausserordentlich schnell verflüssigt und in Dextrin und Zucker übergefübrt wird. Bei anderen Geschöpfen, namentlich bei Fleischfressern, besitzt der Mundspeichel diese Eigenschaft nicht ${ }^{1}$ ). Es ist weiter bekannt, dass jedes Ferment, also auch das Ptyalin des menschlichen Mundspeichels, durch bestimmte Agentien in seiner Wirkung unterstützt, durch andere gehemmt wird. Wärme bis zu gewissen Graden fördert, Kälte setzt beispielsweise die Thätigkeit der meisten Fermente herab. Aehnlich wirken auch Salze, Säuren und Alkalien.

Da über die Wirkungen der letztgenannten Stoffe auf den Mundspeichel nur wenige, zum Theil nicht übereinstimmende Untersuchungen vorliegen, unternahm ich es gern, auf Anregung und unter Anleitung meines verehrten Lehrers, Herrn Prof. Grützner, diese Frage zu bearbeiten.

Nachstehende Untersuchungen sind im physiologischen Institut in Tübingen (mit einer grösseren Unterbrechung) seit Herbst 1897 bis Frühjahr 1899 angestellt worden.

\section{Geschichtliches.}

Die Angaben darüber, in welcher Reaction der Mundspeichel am besten auf gequollene Stärke einwirkt, lauten ausserordentlich

1) Grützner, Pflüger's Archiv für Physiologie Bd. 12 S. 285. 1876. 
verschieden. Die einen sind der Meinung, dass eine neutrale Reaction die beste sei. Dies behaupten z. B. Langley und Eves ${ }^{1}$ ), wiewohl sie auch hin und wieder eine Beförderung der Ptyalinwirkung sahen, wenn genau neutralisirter Speichel mit geringen Mengen von Säuren versetzt wurde. Auch Tigerstedt ${ }^{2}$ ) spricht sich in ähnlichem Sinne wie die genannten beiden Forscher aus.

Andere $^{3}$ ) - und diese Angabe dürfte wohl die verbreitetste sein - halten die alkalische Reaction für diejenige, welche die Verzuckerung am meisten befördere, da ja auch der Speichel selbst in der Regel diese Reaction zeige.

Schliesslich wird namentlich von neueren Forschern noch die Ansicht verfochten, dass ejne schwach saure Reaction bei weitem am kräftigsten die Wirkung des Ptyalins unterstütze. So viel ich

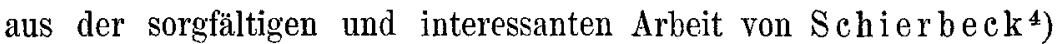
ersehe, der wesentlich diese Behauptung des Näheren für die Kohlensäure begründet hat, waren es Astaschewsky, Chittenden, Griswold und Ely, die als die Ersten dahingehende Angaben machten. Aeltere ähnliche Versuche, in wie weit z. B. künstlich dem Speichel zugesetzter Magensaft die Verzuckerung der. Stärke beeinflusst, finden wir in Lehmann's Zoochemie zusammengestellt.

Uebereinstimmend aber wird von allen Forschern angegeben, dass irgendwie stärkere Säuren oder Alkalien, seien dies fixe oder kohlensaure, die Wirkung des Ptyalins unmöglich machen und das Ferment zerstören. Namentlich konnte Langley ${ }^{5}$ ) in seinen sorgfältigen Untersuchungen zeigen, dass eine Salzsäuremischung, wie sie sich im Magensaft findet, die Wirkung des Speichels aufhebt. Die Frage freilich, $o b$ und wie lange etwa im Magen noch Verzuckerung der Stärke stattfinde, wird verschieden beantwortet.

Was dann schliesslich die Wirkung von Neutralsalzen auf die Speichelwirkung anlangt, so sind mir ausser verschiedenen Notizen in Lehrbüchern ${ }^{6}$ ) nur die Arbeiten von $\mathrm{N}$ asse und von Chittenden

1) Journal of physiology vol. 4 p. 18.1882.

2) Lehrbuch der Physiologie 1897 S. 222.

3) Siehe hierüber Lehmann, Zoochemie 1858 S. 21 und die neueren Lehrbücher der Physiologie, z. B. Hermann's Handbuch Bd. 5 H. 2 S. 33. 1881.

4) Scandinav. Archiv für Physiologie Bd. 3 S. 344 (363). 1892.

5) Journal of physiology vol. 3 p. 246.1882.

6) Hermann's Handbuch Bd. 5 Th. 2 S. 33 ; Landois, Lehrbuch u. s. w. 1896 S. 277 und Hammarsten, Lehrbuch der physiol. Chemie 1891 S. 143. 
und Ely bekannt. Nasse ${ }^{1}$ ) fand bei seinen Versuchen, die er mit menschlichem Mundspeichel unternahm, eine Förderung der Verzuckerung des Stärkekleisters in einer 3,85 procentigen Kochsalzlösung; in einem 7,7 procentigen Kochsalzgemisch einmal eine Hemmung, ein anderes Mal, als mehr Stärkekleister zersetzt werden sollte, dagegen eine Förderung. Die Concentration und die Art der Stärke ist in diesen Versuchen nicht angegeben. Auch andere Natron- und Ammoniaksalze wirkten in einer 4 procentigen Mischung fördernd.

Chittenden und Ely ${ }^{2}$ ) schliesslich erwähnen gelegentlich in ihrer Arbeit über die fördernde Wirkung der Peptone auf das Ptyalin, dass schwache Lösungen von Kochsalz und phosphorsaurem Natron $\left(\mathrm{Na}_{2} \mathrm{HPO}_{4}\right)$ eine ähnliche fördernde Wirkung haben.

\section{Eigene Untersuchungen.}

1. Methodisches.

Zunächst musste ich darauf ausgehen, eine möglichst einfache und genaue Methode zu finden, welche es mir gestattete, den Grad der diastatischen Wirkung nicht bloss in 2 oder 3 Stärkegemischen, sondern in einer bei weitem grösseren Zahl in möglichst kurzer Zeit festzustellen. Denn es kam mir wesentlich darauf an, nicht bloss verschiedene Salze, sondern auch verschiedene Concentrationen derselben Salze mit einander und zwar gleichzeitig miteinander zu vergleichen. Nach einigem Herumprobieren ergab sich mir für die Zuekerbestimmung als die bei weitem einfachste und völlig zureichende Methode die Kaliprobe von Moore, bezw. Heller.

Es wurden alle mit Stärke und Speichel beschickten Reagenzgläser nach einer gewissen Zeit der Einwirkungsdauer des Fermentes mit gleichen Mengen Kalilauge versetzt und, so wie es bereits $\operatorname{Paschutin}^{3}$ ) gethan hatte, in ein grösseres Gefäss mit kochendem Wasser gestellt. Das lästige Stossen, wie es bei directem Kochen nur schwer zu vermeiden ist, wurde hierdurch umgangen; auch war die Einwirkung der Hitze auf jedes einzelne Gläschen von gleicher Dauer und Stärke. Je nach der Menge des gebildeten Zuckers zeigten die verschiedenen Proben eine hellgelbe bis braungelbe Färbung.

1) Pflüger's Archiv Bd. 11 S. 138. 1875.

2) Journal of physiology vol. 3 p. 327.1882.

3) Archiv für Anatomie, Physiologie u. s. w. 1871 S. 305. 
Um die Färbung ihrer Stärke nach abschätzen zu können, verfubr ich ähnlich wie Gr ützner ${ }^{1}$ ) bei seiner colorimetrischen Pepsinbestimmung. Es wurden pämlich yon einer 1 procentigen Kalibichromat-Lösung Verdünnungen hergestellt, so dass in dem Gläschen, das mit 1 bezeichnet wurde, sich die färbende Flüssigkeit zu der Wassermenge verhielt, wie 1:128,

$$
\begin{aligned}
& \text { im Gläschen } 2,1: 64 \text {, } \\
& 4,1: 32 \text {, } \\
& 8,1: 16 \text {, } \\
& 16,1: \text { 8. Der Unterschied in der }
\end{aligned}
$$

Färbung der Gläschen war sehr bedeutend und stimmte namentlich in den belleren Nüancen mit der gelben Farbe der durch Kalilauge umgeänderten Zuckerstärkelösungen gut überein. Auch behielten die Farbegläschen, wenn man sie sorgfältig verschloss, ibre Farbe lange Zeit völlig unverändert, was ausserordentlich vortheilhaft war.

Wurde also beispielsweise bei einem Versuch ein mit Stärke und Speichel versetztes Gläschen nach der Kaliprobe in seiner Färbung dem Farbengläschen 1 gleichgefunden, ein zweites gleich dem Gläschen 4, so wusste man, dass in dem zweiten Gläschen ungefähr 4mal mehr Zucker enthalten war. So liess sich sehr schnell und sicher die ungefähre Menge des gebildeten Zuckers abschätzen; namentlich kounte man die oberen und unteren Farbengrenzen übersehen. Denn wenn ein Verdauungsgläsehen nicht mit der Farbe eines Farbengläschens übereinstimmte, so konnte man doch stets sagen, zwischen welchen zwei Farbengläschen es sich befand ${ }^{2}$ ).

Andere einfachere Methoden, etwa die Filtrationsmethode von Grützner ${ }^{3}$ ) anzuwenden, empfahl sich desshalb nicht, weil namentlich grössere Salzmengen auf die Fluidität und demnach auch auf die Filtrierbarkeit der Stärkegemische einen störenden Einfluss aus-

1) Neue Untersuchungen über Bildung und Ausscheidung des Pepsins. Breslau 1875.

2) Da ich bei meinen ersten Versuchen die erwähnte Farbenscala noch nicht hatte, sondern die Farbenwerthe nur ungefähr bestimmte, habe ich mir nachträgiich erlaubt, die Farbenwerthe der ersten Versuche auch auf die Kalibichromat-Farbenscala zu übertragen, so dass alle Versuche, wenn auch nicht ganz genau, so doch annähernd genau mit einander verglichen werden können. Die nachträglich zugesetzten Nummern der Farben sind in den Versuchstabellen eingeklammert.

3) Pflüger's Archiv Bd.. 12 S. 285 (298). 1875. 
übten. Auch die Trommer'sche, bezw. Fehling'sche Methode, oder andere ähnliche Methoden, konnte ich nicht anwenden, einmal, weil sie bei der Menge der zu untersuchenden Gläschen viel zu umständlich und ausserdem nicht ganz eindeutig gewesen wäre. Denn ich fand, dass bei einer stärkeren Salzconcentration ein starker flockiger Niederschlag entstand und die Ausfällung des Kupferoxyduls auch bei gleich viel Zuckergehalt durch die verschiedenen Salzmengen in unliebsamer Weise gestört wurde.

Hingegen konnte ich mich durch eine Reihe von Versuchen davon überzeugen, dass die von mir angewendeten Zusätze von Salzen, Basen oder Säuren zu Stärkezuckerlösungen keine Beeinträchtigung der Farbe bei der Kaliprobe hervorriefen. Die gleichen Zuckermengen lieferten unter sonst gleichen Bedingungen die gleichen Farbentöne. Folgende Versuche, welche jedesmal bei einem neu zu untersuchenden Reagens als Vorversuche angestellt wurden, mögen den Beweis dafür liefern.

\section{Vorversuch.}

Von einer 4 procentigen Stärkekleisterlösung, welche durch Einwirkung von Speichel theilweise in Zucker umgewandelt worden war, werden je $5 \mathrm{ccm}$ in vier verschiedene Gläschen gegeben und diese in kochendes Wasser gestellt, um die Ptyalinwirkung aufzuheben und das Ferment zu zerstören. Dann werden die Gläschen mit verschiedenen Mengen von Kochsalz versetzt, so dass Gläschen 1 enthält $5 \mathrm{ccm}$ Stärkezuckergemisch $+5 \mathrm{ccm}$ Wasser,

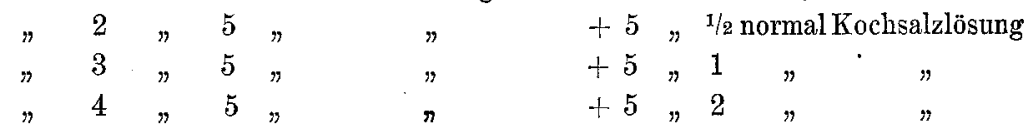

Sodann erhält jedes Gläschen $2 \mathrm{ccm}$ Kalilauge $(10 \%$ ), worauf alle Gläschen in kochendes Wasser gestellt werden. Es zeigte sich, dass alle Gläschen gleich dunkelgelb gefärbt waren; ein Unterschied unter den einzelnen Gläschen in der Farbe war nicht vorhanden. Derselbe Versuch wurde mit Jod-, Brom- und Fluornatrium und -Kalium ebenfalls angestellt, mit demselben Erfolg. Es ergab sich also, dass die Verbindungen des Natriums und des Kaliums die Farben bei der Mo ore'schen Zuckerprobe nicht störend beeinflussten. Ganz ähnliche Versuche wurden mit den Basen und Säuren, die untersucht worden, angestellt, wobei nirgends eine Störung der Moore'schen Probe beobachtet werden konnte.

Die Menge der umgesetzten Stärke aus der noch übrig gebliebenen Stärke, etwa mit der Jodreaction, zu bestimmen, sowie es Langley gethan, wäre wohl möglich, jedoch nicht einfach genug gewesen. Denn es war immer noch so viel Stärke, auch nach längerer Finwirkungszeit des Fermentes (20 Minuten) vorhanden, dass ohne be- 
Ueber die Einwirkung verschiedener chem. Stoffe auf die Thätigkeit etc. 281

sondere Maassnahmen, wie Verdünnungen u. s. w., alle Gläschen nach Jodzusatz eine gleiche, tiefblaue Farbe zeigten. Ausserdem wurde auch hier bei den stärkeren Salzconcentrationen ein Niederschlag von Jodstärke beobachtet, der die Probe nicht unwesentlich störte.

Als zu zersetzendes Object benutzte ich gewöhnlich Weizenstärkekleister von $2 \%$; doch ist bei jedem Versuch die Concentration der Stärke besonders angegeben, da die Verzuckerung derselben in hohem Maasse von ihrer Concentration und Bereitungsart abhängig ist.

Als diastatisches Ferment verwendete ich regelmässig eine Mischung meines Mundspeichels, der mit 1 Theil Wasser versetzt, filtrirt und mit 3 Theilen Glycerin gemischt wurde. Diese Mischung ist ausserordentlich leicht herzustellen und weil lange Zeit haltbar und sehr wirksam, wohl zu empfehlen. Gelegentlich kam auch das Extract aus einer Kaninchenparotis zur Verwendung. Das von einer bekannten Firma bezogene, in Glycerin gelöste „Ptyalin“ stellte eine bräunliche Flüssigkeit dar und erwies sich im Vergleich zu dem selbst hergestellten als fast unwirksam.

Die Reaction des Stärkekleisters, der zu jedem Versuch frisch hergestellt wurde, und die Reaction des Speichelgemisches war immer gegenüber empfindlichem Lakmuspapier neutral.

Von den Salzen verwendete ich der Einfachheit halber die Halogenverbindungen des Natriums und des Kaliums. Was num weiter die Concentration anlangt, so ist es nach den von Grützner ${ }^{1}$ ) angestellten Erwägungen selbstverständlich, dass ich nur äquimoleculare Mengen der genannten Salze miteinander verglichen habe. Würde man, was ich nebenbei bemerke, äquiprocentuale Lösungen in ihren Wirkungen vergleichen, so kämen ganz andere, aber in keinem innern Zusammenhang stehende Thatsachen heraus.

2. Wirkung des Kochsalzes auf die Umwandlung der Stärke durch speichel.

Wie schon oben erwähnt, gibt $\mathrm{O}$. N asse an, dass Kochsalz in einer Concentration von 7,7\% einmal einen hemmenden, das andere Mal einen fördernden Einfluss auf die Verzuckerung seines Stärkekleisters ausgeübt habe. Meine ersten nach dieser Richtung hin angestellten Versuche, von denen ich einige Beispiele folgen lasse, ergaben mir ebenfalls sehr schwankende Resultate. So zeigte sich

1) Grützner, Deutsche medicinische Wochenschrift $1893 \mathrm{Nr} .52$ und Pflüger's Archiv für Physiologie Bd. 53 S. 83, 1893. u. a. 
z. B. nach Versuch 1 und 2, dass schwächere Lösungen als $\frac{1}{1}$ Normallösung von $\mathrm{NaCl}(5,8 \%)$ immer befördern, eine doppelt normale Lösung aber einmal förderte, das zweite Mal entschieden hemmte.

Bei Versuch 1 will ich die Art und Weise, wie bei allen Versuchen vorgegangen wurde, genau beschreiben, um dann bei den späteren Versuchen nur noch durch eine Uebersicht die Concentrationen der Stärke und Salze (oder Säuren und Basen), die Menge des Speichelgemisches und die Menge des gebildeten Zuckers resp. Farbe des Verdauungsgemisches kurz anzugeben.

\section{Versuch 1.}

In 6 gleichweite Reagenzglüschen werden je 5 com einer etwa 6 procentigen Stärkekleisterlösung geschüttet. In jedes Gläschen kommen je 5 ccm verschieden concentrirter Kochsalzlösung. Dieses Gemenge wird dann gut umgeschüttelt, wobei jedes Gläschen seine Nummer vorher erhalten hat. Dans erhält jedes Gemisch einen Zusatz von $0,3 \mathrm{ccm}$ obiger Speichelmischung, worauf wieder um. geschüttelt wird. Nun werden die Gläschen in einem Ständer bei Zimmertemperatur $\left(20^{\circ}\right.$ C.) eine bestimmte Zeit $(5-20$ Minuten) der Einwirkung des Ptyalins ausgesetzt, wobei sie ruhig stehen gelassen werdeu. Nach der bestimmten Zeit, gewöhnlich nach 10 Minuten, erhält jedes Gläschen $2 \mathrm{~cm}$ einer 11procentigen Kalilauge, worauf sie alle gleichzeitig in ein mit kochendem Wasser angefülltes, grösseres Gefäss verbracht werden. Hier verbleiben sie etwa 2 Minuten lang, während das Wasser weiter kocht, und erhalten durch die Siedetemperatur die specifische gelbe bis braungelbe Färbung. Der Werth der Farbe jedes einzelnen Gläschens wird nun gleich bestimmt und hiernach die Gläschen untereinander verglichen.

\section{Ergebniss ron Versuch 1.}

$\mathrm{Mit} \mathrm{NaCl}(58,4$ Moleculargewicht) und Stärke von $3 \%$.

\begin{tabular}{|c|c|c|c|c|c|c|}
\hline 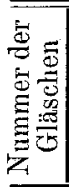 & 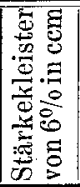 & $\begin{array}{l}\mathrm{NaCl} \text { - bezw. } \\
\mathrm{H}_{2} \mathrm{O} \text {-Mengen } \\
\text { 'in ccm }\end{array}$ & 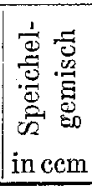 & $\begin{array}{l}\text { Nor- } \\
\text { mal- } \\
\text { Salzge } \\
\text { Miscl }\end{array}$ & $\begin{array}{l}\text { Procen- } \\
\text { tual- } \\
\text { halt der } \\
\text { hungen }\end{array}$ & $\begin{array}{c}\text { Erzielter Farbenton } \\
\text { resp. Zuckergehalt } \\
\text { nach } 10 \text { Minuten }\end{array}$ \\
\hline 1 & 5 & $5 \mathrm{H}_{2} \mathrm{O}$ & 0,3 & 0 & 0 & hellgelb (4) \\
\hline 2 & - & $5 \mathrm{NaCl} \frac{1}{4}$ normal & - & $\frac{1}{8} n$ & $0,7 \%$ & braungelb (10) \\
\hline 3 & - & $5 \mathrm{NaCl} \frac{1}{2}$ normal & - & $\frac{1}{4} n$ & $1,5 \%$ & $\begin{array}{c}\text { braungelb (9) } \\
\text { weniger als Gläschen } 2\end{array}$ \\
\hline 4 & - & $5 \mathrm{NaCl} \frac{1}{1}$ nomal & - & $\frac{1}{2} n$ & $2,9 \%$ & $\begin{array}{l}\text { dunkelgelb (7) } \\
\text { heller als Gläschen } 3\end{array}$ \\
\hline 5 & - & $5 \mathrm{NaCl} \frac{2}{1}$ normal & 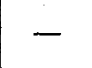 & $\frac{1}{1} n$ & $5,8 \%$ & gelb (5) \\
\hline 6 & - & $5 \mathrm{NaCl} \frac{4}{1}$ normal & - & $\frac{2}{1} \mathrm{n}$ & $11,6 \%$ & $\begin{array}{l}\text { gelb }\left(4^{1 / 2} / 2\right) \text {, heller als } \\
\left.\text { Gl.5, dunkler als Gl. } 1^{1}\right)\end{array}$ \\
\hline
\end{tabular}

1) Betreffs der eingeklammerten Zahlen siehe oben Anmerkung auf 
Wie aus den Farben dieses Versuches leicht zu entnehmen ist, haben alle Kochsalzconcentrationen die Umsetzung des 3 procentigen Stärkekleisters in Zucker begünstigt, bei weitem am meisten aber Lösungen von $0,7-1,5 \%$.

Der folgende Versuch ist im Ganzen in gleicber Weise angestellt worden, nur wurde Stärkekleister von $4 \%$ genommen, so dass also nach Zusatz der Salz- bezw. Wassermenge die Gemische 2 procentige Stärke enthielten.

\section{Versuch 2.}

Mit $\mathrm{NaCl}$ und Stärke von $2 \%$.

\begin{tabular}{|c|c|c|c|c|c|c|c|}
\hline 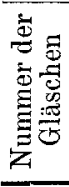 & 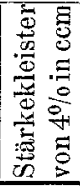 & $\begin{array}{l}\mathrm{NaCl} \text { - bezw. } \\
\mathrm{H}_{2} \mathrm{O} \text {-Mengen } \\
\text { in ccm }\end{array}$ & 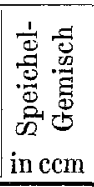 & $\begin{array}{c}\text { Nor- } \\
\text { mal- } \\
\text { Salzg } \\
\text { Mis }\end{array}$ & $\begin{array}{l}\text { Procen- } \\
\text { tual- } \\
\text { alt der } \\
\text { ungen }\end{array}$ & \multicolumn{2}{|c|}{$\begin{array}{c}\text { Erzielter } \\
\text { Farbenton resp. } \\
\text { Zuckergehalt } \\
\text { nach } 10 \text { Minuten }\end{array}$} \\
\hline 1 & 5 & $5 \mathrm{H}_{2} \mathrm{O}$ & 0,3 & 0 & 0 & gelb (5) & \\
\hline 2 & - & $5 \mathrm{NaCl} \frac{1}{4}$ normal & - & $\frac{1}{8} n$ & $0,7 \%$ & braungelb (12) & \\
\hline 3 & - & $5 \mathrm{NaCl} \frac{1}{2}$ normal & - & $\frac{1}{4} \mathrm{n}$ & $1,5 \%$ & braungelb (11) & 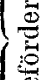 \\
\hline 4 & - & $5 \mathrm{NaCl} \frac{1}{1}$ normal & - & $\frac{1}{2} n$ & $2,9 \%$ & dunkelgelb (7) & 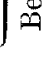 \\
\hline 5 & - & $5 \mathrm{NaCl} \frac{2}{1}$ normal & - & $\frac{1}{1} \mathbf{n}$ & $5,8 \%$ & hellgelb $\left(4^{1 / 2}\right)$ & 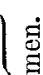 \\
\hline 6 & - & $5 \mathrm{NaCl} \frac{4}{\mathrm{l}}$ normal & - & $\frac{2}{1} \mathrm{n}$ & $11,6 \%$ & hellgelb (4) & $\stackrel{\text { gี }}{=}$ \\
\hline
\end{tabular}

Bei 2 procentiger Stärke hat eine Kochsalzconcentration von $5,8 \%$ die Verzuckerung schon deutlich gehemmt, die schwächeren aber haben sie nach wie vor befördert.

Ehe ich zu den in den beiden obigen Versuchen besprochenen Ergebnissen gelangte, machte ich eine grosse Reihe von Versuchen, die mir, obwohl ich mir keines Fehlers beim Arbeiten bewusst war, doch fortwährend schwankende und wechselnde Resultate lieferten. Bald wirkte dieselbe Salzconcentration hemmend, bald förderte sie. Der einzige Unterschied der betreffenden Versuche bestand, worauf ich anfänglich, ebenso wie wahrscheinlich $\mathrm{N}$ asse nicht genau achtete, in der verschiedenen Concentration der Stärkelösungen. Erst später kam ich bei meinen Versuchen mit Salzsäure auf die bemerkenswerthe Thatsache, dass die Ptyalinwirkung nicht bloss abhängig ist von den Salz- oder Säuremolecülen, bezw. ihren disso- 
ciirten Bestandtheilen, sondern in zweiter Linie auch abhängig ist von der Menge der Stärkemolecüle. Je concentrirter nämlich die Stärke ist, um so concentrirter können auch die Salzlösungen sein, ehe sie hemmend wirken; oder anders ausgedrückt: Kochsalzlösungen derselben Concentration wirken unter sonst gleichen Bedingungen hemmend auf die Verzuckerung dünner Stärkegemische, fördernd auf die Verzuckerung dicker Stärkegemische. Hiermit lösen sich leicht meine, so wie $\mathrm{Nasse}$ 's widersprechende Angaben.

Zum Beweise dieser soeben aufgestellten Sätze lasse ich einige weitere Versuche folgen.

\section{Versuch 3.}

Mit $\mathrm{NaCl}$ und Stärke von $4 \%$.

\begin{tabular}{|c|c|c|c|c|c|c|c|}
\hline $\begin{array}{l}\text { Nummer } \\
\text { der } \\
\text { Gläschen }\end{array}$ & $\begin{array}{l}\text { Stärke- } \\
\text { kleister } \\
\text { von } 8 \% \\
\text { in ccm }\end{array}$ & $\begin{array}{l}\mathrm{NaCl}-\text { bezw. } \\
\mathrm{H}_{2} \mathrm{O} \text {-Mengen } \\
\text { in ccm }\end{array}$ & $\begin{array}{l}\text { Speichel- } \\
\text { gemisch } \\
\text { in ccm }\end{array}$ & \multicolumn{2}{|c|}{$\begin{array}{l}\text { Salzgehalt der } \\
\text { Mischungen }\end{array}$} & \multicolumn{2}{|c|}{$\begin{array}{c}\text { Erzielter } \\
\text { Farbenton resp. } \\
\text { Zuckermenge } \\
\text { nach10 Minuten }\end{array}$} \\
\hline 1 & 5 & $5 \mathrm{H}_{2} \mathrm{O}$ & 0,3 & 0 & 0 & 2 & \\
\hline 2 & - & $5 \mathrm{NaCl} \frac{1}{64}$ normal & - & $\frac{1}{128} \mathrm{n}$ & $0,045^{\circ} \%$ & 6 & \\
\hline 3 & - & $5 \mathrm{NaCl} \frac{1}{32}$ normal & - & $\frac{1}{64} \mathrm{n}$ & $0,09 \%$ & 6 & \\
\hline 4 & - & $5 \mathrm{NaCl} \frac{1}{16}$ normal & - & $\frac{1}{32} \mathrm{n}$ & $0,17 \%$ & $4^{1 / 2}$ & \\
\hline 5 & - & $5 \mathrm{NaCl} \frac{1}{8}$ normal & - & $\frac{1}{16} \mathrm{n}$ & $0,35 \%$ & 4 & 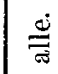 \\
\hline 6 & - & $5 \mathrm{NaCl} \frac{1}{4}$ normal & - & $\frac{1}{8} n$ & $0,7 \%$ & $3^{1 / 2}$ & 递 \\
\hline 7 & - & $5 \mathrm{NaCl} \frac{1}{2}$ normal & 一 & $\frac{1}{4} \mathrm{n}$ & $1,5 \%$ & 3 & ڤ. \\
\hline 8 & - & $5 \mathrm{NaCl} \frac{1}{1}$ normal & - & $\frac{1}{2} \mathrm{n}$ & $2,9 \%$ & $2^{1 / 2}$ & \\
\hline 9 & - & $5 \mathrm{NaCl} \frac{2}{1}$ normal & - & $\frac{1}{1} \mathrm{n}$ & $5,8 \%$ & $2^{1 / 2}$ & \\
\hline 10 & - & $5 \mathrm{NaCl} \frac{4}{1}$ normal & - & $\frac{2}{1} \mathrm{n}$ & $11,6 \%$ & $21 / 4$ & \\
\hline
\end{tabular}

Bei Stärke von $4 \%$ befördert sogar eine Kochsalzlösung von $11,6 \%$ die Verzuckerung noch deutlich. Es ist ferner bemerkenswerth, in welch' bedeutender Weise selbst die ganz schwachen Kochsalzlösungen die Fermentwirkung unterstützen. 
Ueber die Einwirkung verschiedener chem. Stoffe auf die Thätigkeit etc. 285

Versuch 4.

Mit $\mathrm{NaCl}$ und Stärke ron $2 \%$.

\begin{tabular}{|c|c|c|c|c|c|c|c|}
\hline $\begin{array}{c}\text { Nummer } \\
\text { der } \\
\text { Gläschen }\end{array}$ & \begin{tabular}{|l|} 
Stärke- \\
kleister \\
von $4 \%$ \\
in ccm
\end{tabular} & $\begin{array}{l}\mathrm{NaCl} \text { bezw. } \\
\mathrm{H}_{2} \mathrm{O} \text {-Mengen } \\
\text { in ccm }\end{array}$ & $\begin{array}{l}\text { Speichel- } \\
\text { gemisch } \\
\text { in ccm }\end{array}$ & $\begin{array}{l}\text { Nor- } \\
\text { mal- } \\
\text { Salzo } \\
\text { Mis }\end{array}$ & $\begin{array}{l}\text { Procen- } \\
\text { tual- } \\
\text { halt der } \\
\text { hungen }\end{array}$ & \multicolumn{2}{|c|}{$\begin{array}{c}\text { Erzielter } \\
\text { Farbenton } \\
\text { nach } 10 \mathrm{Min} .\end{array}$} \\
\hline 1 & 5 & $5 \mathrm{H}_{2} \mathrm{O}$ & 0,3 & 0 & 0 & 8 & \\
\hline 2 & - & $5 \mathrm{NaCl} \frac{1}{64}$ normal & - & $\frac{1}{128} \mathrm{n}$ & $0,045 \%$ & 9 & \\
\hline 3 & 一 & $5 \mathrm{NaCl} \frac{1}{32}$ normal & - & $\frac{1}{64} n$ & $0,09 \%$ & 10 & \\
\hline 4 & - & $5 \mathrm{NaCl} \frac{1}{16}$ normal & - & $\frac{1}{32} n$ & $0,17 \%$ & 9 & ن \\
\hline 5 & - & $5 \mathrm{NaCl} \frac{1}{8}$ normal & - & $\frac{1}{16} \mathrm{n}$ & $0,35 \%$ & 8 & 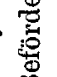 \\
\hline 6 & - & $5 \mathrm{NaCl} \frac{1}{4}$ normal & - & $\frac{1}{8} n$ & $0,7 \%$ & 7 & \\
\hline 7 & - & $5 \mathrm{NaCl} \frac{1}{2}$ normal & - & $\frac{1}{4} \mathrm{n}$ & $1,5 \%$ & 5 & \\
\hline 8 & - & $5 \mathrm{NaCl} \frac{1}{1}$ normal & - & $\frac{1}{2} \mathrm{n}$ & $2,9 \%$ & 3,5 & \\
\hline 9 & - & $5 \mathrm{NaCl} \frac{2}{1}$ normal & - & $\frac{1}{1} n$ & $5,8 \%$ & 2 & 覀 \\
\hline 10 & - & $5 \mathrm{NaCl} \frac{4}{1}$ normal & - & $\frac{2}{1} \mathrm{n}$ & $11,6 \%$ & 2 & हु \\
\hline
\end{tabular}

Bei Stärkegemischen von $2 \%$ ist der hemmende Einfluss des Kochsalzes schon deutlich bei 5,8\% Salzgehalt. Schwache Lösungen dagegen befördern nach wie vor sehr bedeutend.

\section{Yersuch 5.}

Mit $\mathrm{NaCl}$ und Stärke von $1 \%$.

\begin{tabular}{c|c|c|c|c|c|c}
\hline \hline $\begin{array}{c}\text { Nummer } \\
\text { der } \\
\text { Gläschen }\end{array}$ & $\begin{array}{c}\text { Stärke- } \\
\text { kleister } \\
\text { von 2\% } \\
\text { in ccm }\end{array}$ & $\begin{array}{c}\text { NaCl - bezw. } \\
\mathrm{H}_{2} \mathrm{O} \text {-Mengen } \\
\text { in ccm }\end{array}$ & $\begin{array}{c}\text { Speichel- } \\
\text { gemisch } \\
\text { in ccm }\end{array}$ & $\begin{array}{c}\text { Nor- } \\
\text { mal- } \\
\text { Salzgehalt der } \\
\text { Nischungen }\end{array}$ & $\begin{array}{c}\text { Procen- } \\
\text { tual- }\end{array}$ & $\begin{array}{c}\text { Erzielter } \\
\text { Farbenton } \\
\text { nach 10 Min. }\end{array}$ \\
\hline 1 & 5 & $5 \mathrm{H}_{2} \mathrm{O}$ & 0,3 & 0 & 0 & 5 \\
2 & - & $5 \mathrm{NaCl} \frac{1}{64}$ normal & - & $\frac{1}{128} \mathrm{n}$ & $0,045 \%$ & 12 \\
3 & - & $5 \mathrm{NaCl} \frac{1}{32}$ normal & - & $\frac{1}{64} \mathrm{n}$ & $0,09 \%$ & 13 \\
4 & - & $5 \mathrm{NaCl} \frac{1}{16}$ normal & - & $\frac{1}{32} \mathrm{n}$ & $0,17 \%$ & 11 \\
5 & - & $5 \mathrm{NaCl} \frac{1}{8}$ normal & - & $\frac{1}{16} \mathrm{n}$ & $0,35 \%$ & 9 \\
6 & - & $5 \mathrm{NaCl} \frac{1}{4}$ normal & - & $\frac{1}{8} \mathrm{n}$ & $0,7 \%$ & 7
\end{tabular}


(Fortsetzung.)

$\left.\begin{array}{c|c|c|c|c|c|c}\hline \begin{array}{c}\text { Nummer } \\ \text { der }\end{array} & \begin{array}{c}\text { Stärke- } \\ \text { kleister } \\ \text { von 2\% }\end{array} & \begin{array}{c}\text { NaCl- bezw. } \\ \mathrm{H}_{2} \text { O-Mengen } \\ \text { in ccm ccm }\end{array} & \begin{array}{c}\text { Speichel- } \\ \text { gemisch } \\ \text { in ccm }\end{array} & \begin{array}{c}\text { Nor- } \\ \text { mal- } \\ \text { Salzgehalt der } \\ \text { Mischungen }\end{array} & \begin{array}{c}\text { Procen- } \\ \text { tual- }\end{array} & \begin{array}{c}\text { Erzielter } \\ \text { Farbenton } \\ \text { nach 10 Min. }\end{array} \\ \hline 7 & - & 5 \mathrm{NaCl} \frac{1}{2} \text { normal } & - & \frac{1}{4} \mathrm{n} & 1,5 \% & 4 \\ 8 & - & 5 \mathrm{NaCl} \frac{1}{1} \text { normal } & - & \frac{1}{2} \mathrm{n} & 2,9 \% & 31 / 2 \\ 9 & - & 5 \mathrm{NaCl} \frac{2}{1} \text { normal } & - & \frac{1}{1} \mathrm{n} & 5,8 \% & 3 \\ 10 & - & 5 \mathrm{NaCl} \frac{4}{1} \text { normal } & - & \frac{2}{1} \mathrm{n} & 11,6 \% & 2\end{array}\right\}$

Bei Stärkegemischen von $1 \%$ hemmt schon eine 1,5 procentige Salzconcentration deutlich; schwache befördern ungemein stark.

In Fig. 1, 2 und 3 auf folgender Seite sind die Versuche 3, 4 und 5 in Curven wiedergegeben. Auf der Horizontalen $(0-\mathrm{NaCl})$ sind die Salzconcentrationen verzeichnet. Auf der Senkrechten $(0-8)$ sind die Farbenwerthe der gebildeten Zuckermengen vermerkt. Die Horizontale "St" gibt bei jedem Versuch die Höhe der gebildeten Zuckermenge im Stärkekleister ohne Salzzusatz an. Zwischen $\frac{1}{16}$ und $\frac{1}{8} \mathrm{n}$ bei $a$, ebenso zwischen $\frac{1}{1}$ und $\frac{2}{1} \mathrm{n}$ bei $b$ ist die Curve unterbrochen dargestellt, da sie sich sonst $\mathrm{zu}$ weit ausgedehnt hätte. Ausserdem sind die Curven von $\frac{1}{8}-\frac{1}{1} \mathrm{n}$ aus demselben Grunde stark verkürzt.

Diese Versuche beweisen den oben (S. 284) ausgesprochenen Satz und zejgen zugleich, dass die von mir verwendeten Fermentmengen innerhalb derselben Zeit um so mehr Stärke in Zucker verwandeln, je dünner die Stärkelösungen sind. (Vgl. die Horizontalen $S t$ in Fig. 1, 2 und 3.) Diese Thatsache habe ich regelmässig und ganz ausnahmslos beobachtet, auch noch durch besondere, darauf hingerichtete Versuche erhärtet. So zeigten z. B. in einem derartigen Versuche die Gläschen nach 10 Minuten langer. Einwirkung von je $0,3 \mathrm{~cm}$ Fermentmischung in 4, 2 und 1 procentigen Stärkekleistermischungen, bezw. die Farben 2, 3 und 4.

Offenbar kann in den dünnen Stärkelösungen, in welchen die Stärkemolecüle wohl viel leichter beweglich sind, das Ferment besser zu ihnen beran, als in den dicken. Andererseits schützen 
Ueber die Einwirkung verschiedener chem. Stoffe auf die Thätigkeit etc. 287 aber diese wiederum bei Salzzusatz das Ferment von dem Einfluss dieses Stoffes. Sie wirken gewissermaassen "einhüllend."

$\mathrm{Ob}$ hierbei eine Art chemischer Verbindung oder Bindung der Salz- und Stärkemoleeüle stattfindet, wie Aehnliches von den Eiweiss-

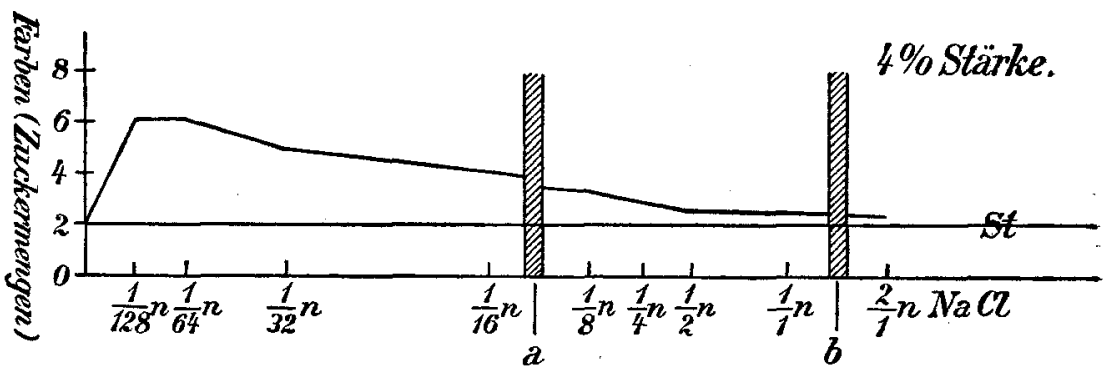

Fig. 1.

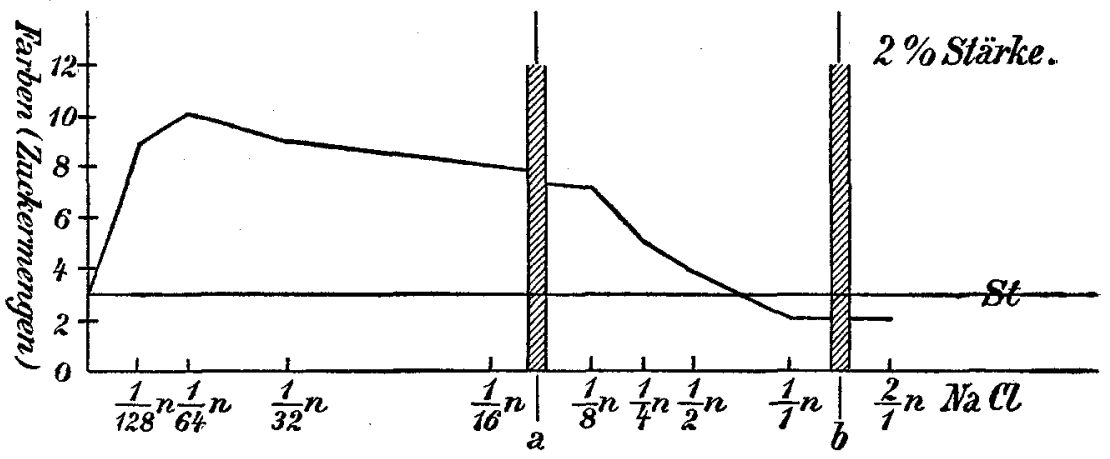

Fig. 2.

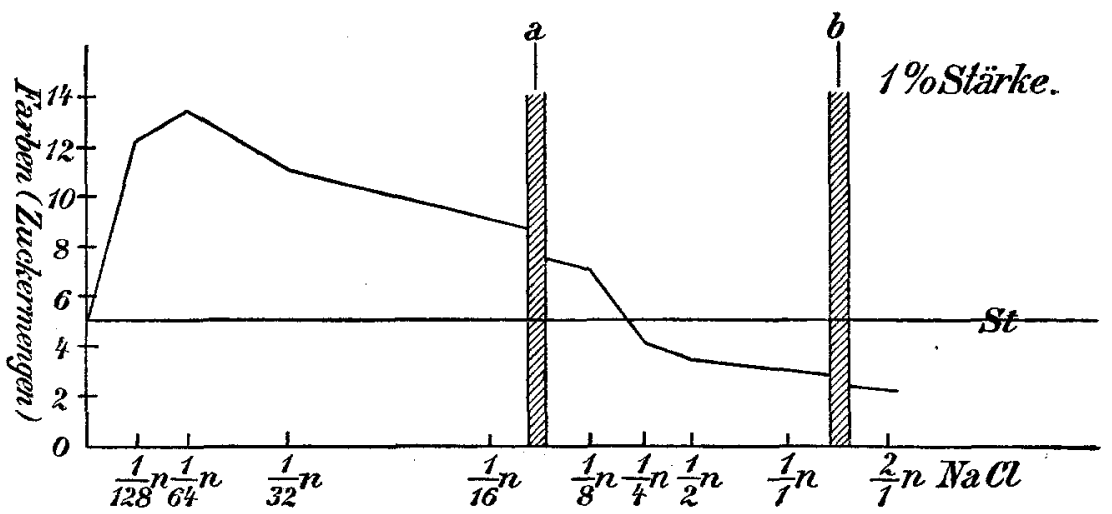

Fig. 3.

E. Pflũ ger, Archiv für Physiologie. Bd. 76. 
körpern, bezw. Peptonen und Säuren ${ }^{1}$ ) bekannt ist; darüber will ich mir ein Urtheil nicht erlauben. Jedenfalls aber haben die beiden Vorgänge ausserordentlich viel Aehnliches:

So weit mir bekannt; hat noch Niemand auf diese Thatsache hingewiesen. Nur in der Arbeit von Paschutin ${ }^{2}$ ) finden sich ähnliche Angaben. Einmal brachte P a s c h u ti n gleiche Fermentmengen mit versehiedenen Mengen von gleich concentrirtem Stärkekleister zusammen und fand, was man wohl voraussehen konnte, eine Bildung von mehr Zucker in denjenigen Gläschen, die dem Volumen nach weniger Stärke, und demnach eine procentisch stärkere Fermentlösung enthielten. Dann aber stellte er einen den unsrigen ähnlichen Versuch an, indem er gleiche Mengen Kleisters von verschiedener Concentration mit gleichen Fermentmengen mischte. Er liess dann in diesem (wie in dem ersten) Versuch das Ferment allerdings nur 20 Secunden einwirken und fand, "dass die Verwandlung desto prägnanter ist, je höher der Procentgehalt des Kleisters war".

Letztere Angabe konnte, obwohl sie von mir und schon vorher von Herrn Prof. Grützner und Andern nachgeprüft wurde, von uns nicht bestätigt werden, auch wenn wir genat nach den Angaben Paschutin's verfuhren. Sowohl bei dieser ausserordentlich kurz dauernden Einwirkung des Speichels (von 20 Secunden), was den Versuch in hohem Maasse ersehwerte, als auch bei einer länger dauernden von 5-10 Minuten, sahen wir stets, dass aus der dickeren Stärke weniger Zucker gebildet wurde als̄ aus der dünneren, genauer gesagt, dass die mit ihr beschickten Gläschen beim Kochen * mit Kalilauge nicht so dunkelgelb wurden, als diejenigen mit der dünneren Stärke.

Was die Ursache des verschiedenen Erfolges bei Paschutin und bei uns anlangt, so kann ich darüber keine bestimmten Angaben machen. Die Art der Fermentes, vielleicht auch die Art und Zubereitung der Stärke können möglicher Weise die verschiedenen Erfolge erklären. Auch hat $\mathrm{P}$ aschutin vielleicht wesentlich nur auf den Anfang der Geibfärbung sein Augenmerk gerichtet, während wir stets die Gläschen erst mit einander verglichen, wenn sie ein paar Minuten im kochenden Wasser gestanden batten.

1) Vgl. hierüber Brücke, Vorlesungen über Physiologie Bd. 1 S. 297, 1874 und die S. 277 erwähnte Arbeit von Langley und Eves.

2) Siehe oben S. 278 und S. 328 in der betreffenden Abhandlung. 
Ueber die Einwirkung verschiedener chem. Stoffe auf die Thätigkeit etc. 289

Bei dem ersten Versuch von $\mathrm{P}$ a schutin hingegen mit gleichen Ferment-, aber verschiedenen Stärkemengen erhielten wir dieselben Ergebnisse, wie jener Forscher; die concentrirte Fermentlösung wirkte stärker, wie Aehnliches auch Grützner ${ }^{1}$ ) bei seinen Untersuchangen über die Wirkung verschieden starker Pepsinlösungen gesehen hatte. Dieselbe Menge Ferment in dünner Lösung (also in einem grossen Volumen Flüssigkeit) wirkte nicht so stark verdauend, als eben so viel Ferment in stärkerer Lồsung (also in einem kleineren Volumen Flüssigkeit). Selbstverständlich gelten alle diese Gesetzlichkeiten, was auch $\mathrm{P}$ aschutin mit Recht hervorhebt, nur innerhalb gewisser, wenn auch sehr weiter Grenzen.

3. Die Wirkungenvon Fluor-, Chlor-, Brom-und Jodnatrium a ufdie Umwandlung von Stärkedureh Speichel mit einander verglichen.

\section{Versuch 6.}

Mit $\mathrm{NaF}(42,0$ Moleculargewicht) und Stärke von $2 \%$.

\begin{tabular}{|c|c|c|c|c|c|c|c|}
\hline $\begin{array}{l}\text { Nummer } \\
: \text { der } \\
\text { Gläschen }\end{array}$ & $\begin{array}{l}\text { Stärke- } \\
\text { kleister } \\
\text { von } 4 \% \\
\text { in ccm }\end{array}$ & $\begin{array}{l}\mathrm{NaF}-\text { bezw. } \\
\mathrm{H}_{2} \mathrm{O}-\text { Mengen } \\
\text { in cem }\end{array}$ & $\begin{array}{l}\text { Speichel- } \\
\text { gemisch } \\
\text { in ccm }\end{array}$ & $\begin{array}{l}\text { Nor- } \\
\text { mal- } \\
\text { Salzg } \\
\text { Mis }\end{array}$ & $\begin{array}{l}\text { Procen- } \\
\text { tual- } \\
\text { alt der } \\
\text { ungen }\end{array}$ & \multicolumn{2}{|c|}{$\begin{array}{c}\text { Erzielter } \\
\text { Farbenton } \\
\text { nach } 10 \mathrm{Min} .\end{array}$} \\
\hline 1 & 5 & $5 \mathrm{H}_{2} \mathrm{O}$ & 0,3 & 0 & 0 & 2 & \\
\hline 2 & - & $5 \mathrm{NaF} \frac{1}{4}$ normal & - & $\frac{1}{8} \mathrm{n}$ & $0,5 \%$ & 7 & \\
\hline 3 & - & $5 \mathrm{NaF} \frac{1}{2}$ normal & - & $\frac{1}{4} \mathrm{n}$ & $1,02 \%$ & 7 & 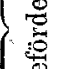 \\
\hline 4 & - & $5 \mathrm{NaF} 1$ rormal & 一 & $\frac{1}{2} \mathrm{n}$ & $2,05 \%$ & 8 & \\
\hline
\end{tabular}

Sämmtliche Concentrationen haben stark befördert, und merkwürdiger Weise die starken besser, als die schwachen. Eine Concentration von $\frac{1}{1}$ normal Salzgehalt konnte nicht mehr versucht werden, weil das Salz sich nur etwa bis $4 \%$ löst.

1) Grützner, Neue Untersuchungen über die Bildung us s. w. des Pepsins S. 14. Breslau 1875. 


\section{Yersuch 7.}

Mit $\mathrm{NaBr}(103,0$ Moleculargewicht) und Stärke von $2 \%$.

\begin{tabular}{c|c|c|c|c|c|c}
\hline \hline $\begin{array}{c}\text { Nummer } \\
\text { der } \\
\text { Gläschen }\end{array}$ & $\begin{array}{c}\text { Stärke- } \\
\text { kleister } \\
\text { von } 4 \% \\
\text { in ccm }\end{array}$ & $\begin{array}{c}\text { NaBr- bezw. } \\
\mathrm{H}_{2} \mathrm{O}-\text { Mengen } \\
\text { in ccm }\end{array}$ & $\begin{array}{c}\text { Speichel- } \\
\text { gemisch } \\
\text { in ccm }\end{array}$ & $\begin{array}{c}\text { Nor- } \\
\text { mal- } \\
\text { Salzgehalt der } \\
\text { Mischungen }\end{array}$ & $\begin{array}{c}\text { Procen- } \\
\text { tual- }\end{array}$ & $\begin{array}{c}\text { Erzielter } \\
\text { Farbenton } \\
\text { nach 10 Min. }\end{array}$ \\
\hline 1 & 5 & $5 \mathrm{H}_{2} \mathrm{O}$ & 0,3 & 0 & 0 & 2 \\
2 & - & $5 \mathrm{NaBr} \frac{1}{4}$ normal & - & $\frac{1}{8} \mathrm{n}$ & $1,27 \%$ & 6 \\
3 & - & $5 \mathrm{NaBr} \frac{1}{2}$ normal & - & $\frac{1}{4} \mathrm{n}$ & $2,5 \%$ & 5 \\
4 & - & $5 \mathrm{NaBr} \frac{1}{1}$ normal & - & $\frac{1}{2} \mathrm{n}$ & $5,0 \%$ & 4
\end{tabular}

Eine $\frac{1}{1}$ normal NaBr-Lösung hemmt stark.

\section{Versuch 8.}

Mit $\mathrm{NaJ}$ (150,0 Moleculargewicht) und Stärke von $2 \%$.

\begin{tabular}{|c|c|c|c|c|c|c|c|}
\hline $\begin{array}{l}\text { Nummer } \\
\text { der } \\
\text { Gläschen }\end{array}$ & $\begin{array}{l}\text { Stärke- } \\
\text { kleister } \\
\text { von } 4 \% \\
\text { in ccm }\end{array}$ & $\begin{array}{l}\mathrm{NaJ} \text {-bezw. } \\
\mathrm{H}_{2} \mathrm{O} \text {-Mengen } \\
\text { in ccm }\end{array}$ & $\begin{array}{l}\text { Speichel- } \\
\text { gemisch } \\
\text { in ccm }\end{array}$ & $\begin{array}{l}\text { Nor- } \\
\text { mal- } \\
\text { Salzg } \\
\text { Mis }\end{array}$ & $\begin{array}{l}\text { Procen- } \\
\text { tual- } \\
\text { alt der } \\
\text { ungen }\end{array}$ & \multicolumn{2}{|c|}{$\begin{array}{c}\text { Erzielter } \\
\text { Farbenton } \\
\text { nach } 10 \text { Min. }\end{array}$} \\
\hline 1 & 5 & $5 \mathrm{H}_{2} \mathrm{O}$ & 0,3 & 0 & 0 & 2 & \\
\hline 2 & - & $5 \mathrm{NaJ} \frac{1}{4}$ normal & - & $\frac{1}{8} \mathrm{n}$ & $1,9 \%$ & 6 & \\
\hline 3 & - & $5 \mathrm{NaJ} \frac{1}{2}$ normal & - & $\frac{1}{4} \mathrm{n}$ & $3,7 \%$ & 5 & \\
\hline 4 & - & $5 \mathrm{NaJ} \frac{1}{1}$ normal & - & $\frac{1}{2} \mathrm{n}$ & $7,5 \%$ & 1,5 & : \\
\hline 5 & - & $5 \mathrm{NaJ} \frac{2}{1}$ normal & - & $\frac{1}{1} \mathrm{n}$ & $15,0 \%$ & 1 & 寻 \\
\hline
\end{tabular}

Wie aus den Farbenwerthen der Versuche $6-9$ leicht zu entnehmen ist, hat unter den 3 Halogenverbindungen des Natriums, die in dem Versuch aufgeführt sind, diejenige des Chlors am besten, diejenige des Jods am wenigsten die Verzuckerung befördert. Dass die Fluorverbindung noch mehr als die Chlorverbindung des Natriums die Saccharification begünstigt, zeigt der folgende Versuch 10, der aus einer Reihe von ähnlichen Versuchen, welche gleiche Resultate ergaben, herausgenommen ist. 
Ueber die Einwirkung verschiedener chem. Stoffe auf die Thätigkeit etc. 291

Versuch 9.

Mit $\mathrm{NaCl}, \mathrm{NaBr}$ und $\mathrm{NaJ}$ und Stärke von $2 \%$.

\begin{tabular}{|c|c|c|c|c|c|c|c|}
\hline $\begin{array}{l}\text { Nummer } \\
\text { der } \\
\text { Gläschen }\end{array}$ & $\begin{array}{l}\text { Stärke- } \\
\text { kleister } \\
\text { von } 4^{0} \% \\
\text { in ccm }\end{array}$ & $\begin{array}{l}\text { Salz- bezw. } \\
\mathrm{H}_{2} \mathrm{O}-\mathrm{M} \text { engen } \\
\text { in ccm }\end{array}$ & $\begin{array}{l}\text { Speichel- } \\
\text { gemisch } \\
\text { in cem }\end{array}$ & $\begin{array}{l}\text { Nor- } \\
\text { mal- } \\
\text { Salzg } \\
\text { Misc }\end{array}$ & $\begin{array}{l}\text { Procen- } \\
\text { tual- } \\
\text { lalt der } \\
\text { ungen }\end{array}$ & \multicolumn{2}{|c|}{$\begin{array}{c}\text { Erzielter } \\
\text { Farbenton } \\
\text { nach } \\
10 \text { Minuten }\end{array}$} \\
\hline 1 & 5 & $5 \mathrm{H}_{2} \mathrm{O}$ & 0,3 & 0 & 0 & 2 & \\
\hline 2 & - & $5 \mathrm{NaCl} \frac{1}{4}$ normal & - & $\frac{1}{8} \mathrm{n}$ & $0,7 \%$ & 7 & \\
\hline 3 & - & $5 \mathrm{NaCl} \frac{1}{2}$ normal & - & $\frac{1}{4} \mathrm{n}$ & $1,4 \%$ & 6 & 苞 \\
\hline 4 & - & $5 \mathrm{NaCl} \frac{1}{1}$ normal & - & $\frac{1}{2} \mathrm{n}$ & $2,9 \%$ & 3,5 & \\
\hline 5 & - & $5 \mathrm{NaCl} \frac{2}{1}$ normal & - & $\frac{1}{1} \mathfrak{n}$ & $5,8 \%$ & 1,5 & 急 \\
\hline 6 & - & $5 \mathrm{NaBr} \frac{1}{4}$ normal & - & $\frac{1}{8} \mathrm{n}$ & $1,27 \%$ & 5,5 & $\dot{\square}$ \\
\hline 7 & - & $5 \mathrm{NaBr} \frac{1}{2}$ normal & - & $\frac{1}{4} \mathrm{n}$ & $2,5 \%$ & 5 & 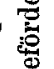 \\
\hline 8 & $\rightarrow$ & $5 \mathrm{NaBr} \frac{1}{1}$ normal & 一 & $\frac{1}{2} \mathrm{n}$ & $5,0 \%$ & 3 & 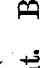 \\
\hline 9 & - & $5 \mathrm{NaBr} \frac{2}{1}$ normal & - & $\frac{1}{1} \mathrm{n}$ & $10,0 \%$ & 1,3 & $\stackrel{\Xi}{\Xi}$ \\
\hline 10 & - & $5 \mathrm{NaJ} \frac{1}{4}$ normal & - & $\frac{1}{8} \mathfrak{n}$ & $1,8 \%$ & 4,5 & 苞 \\
\hline 11 & - & $5 \mathrm{NaJ} \frac{1}{2}$ normal & - & $\frac{1}{4} \mathrm{n}$ & $3,6 \%$ & 4 & की \\
\hline 12 & - & $5 \mathrm{NaJ} \frac{1}{1}$ normal & - & $\frac{1}{2} \mathrm{n}$ & $7,5 \%$ & 1 & छี \\
\hline 13 & - & $5 \mathrm{NaJ} \frac{2}{1}$ normal & - & $\frac{1}{1} n$ & $15,0 \%$ & 0,5 & 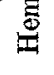 \\
\hline
\end{tabular}

Versuch 10.

Mit $\mathrm{NaF}, \mathrm{NaCl}, \mathrm{NaBr}$ und $\mathrm{NaJ}$ und Stärke von $2 \%$.

\begin{tabular}{|c|c|c|c|c|c|c|c|}
\hline $\begin{array}{c}\text { Nummer } \\
\text { der } \\
\text { Gläschen }\end{array}$ & $\begin{array}{l}\text { Stärke- } \\
\text { kleister } \\
\text { von } 4 \% \\
\text { in } \text { ecm }\end{array}$ & $\begin{array}{l}\text { Salz- bezw. } \\
\mathrm{H}_{2} \mathrm{O} \cdot \text { Mengen } \\
\text { in ccm }\end{array}$ & $\begin{array}{l}\text { Speichel- } \\
\text { gemisch } \\
\text { in } \mathrm{ccm}\end{array}$ & \multicolumn{2}{|c|}{$\begin{array}{c}\text { Salzgehalt der } \\
\text { Mischungen }\end{array}$} & \multicolumn{2}{|c|}{$\begin{array}{c}\text { Erzielter } \\
\text { Farbenton } \\
\text { nach } \\
10 \text { Minuten }\end{array}$} \\
\hline 1 & 5 & $5 \mathrm{H}_{2} \mathrm{O}$ & 0,3 & 0 & 0 & 2 & \\
\hline 2 & - & $5 \mathrm{NaF} 1$ normal & - & $\frac{1}{2} \mathrm{n}$ & $2,05 \%$ & 5 & \\
\hline 3 & - & $5 \mathrm{NaCl} I$ normal & - & $\frac{1}{2} n$ & $2,9 \%$ & 4 & 䒕 \\
\hline 4 & - & $5 \mathrm{NaBr} 1$ normal & - & $\frac{1}{2} \mathfrak{n}$ & $5,0 \%$ & 3 & \\
\hline 5 & - & $5 \mathrm{NaJ} 1$ normal & - & $\frac{1}{2} n$ & $7,5 \%$ & 1 & 峞 \\
\hline
\end{tabular}


In Figur 4 ist die Wirkung der Halogenverbindungen des Natriums, wie sich dieselbe aus den Versuchen 9, 10 und anderen ergibt, dargestellt; es gilt auch für diese Figur das bei den Figuren 1, 22 und 3 schon Gesagte. Die Horizontale in der Höhe von 2 gibt die Menge des gebildeten Zuckers bei Stärke von $2 \%$ ohne Salzzusatz an. Ein Blick auf diese Curven zeigt, wie schwache Salzconcentrationen in hohem Maasse fördern. Ebenso aber zeigt auch die Figur den Unterschied der verschiedenen Salze in ihrer Ein̈wirkung auf die Zuckerbildung durch den Speichel. Das Fluornatrium wirkt am besten, namentlich in verhältnissmässig starken Lösungen, dann folgen

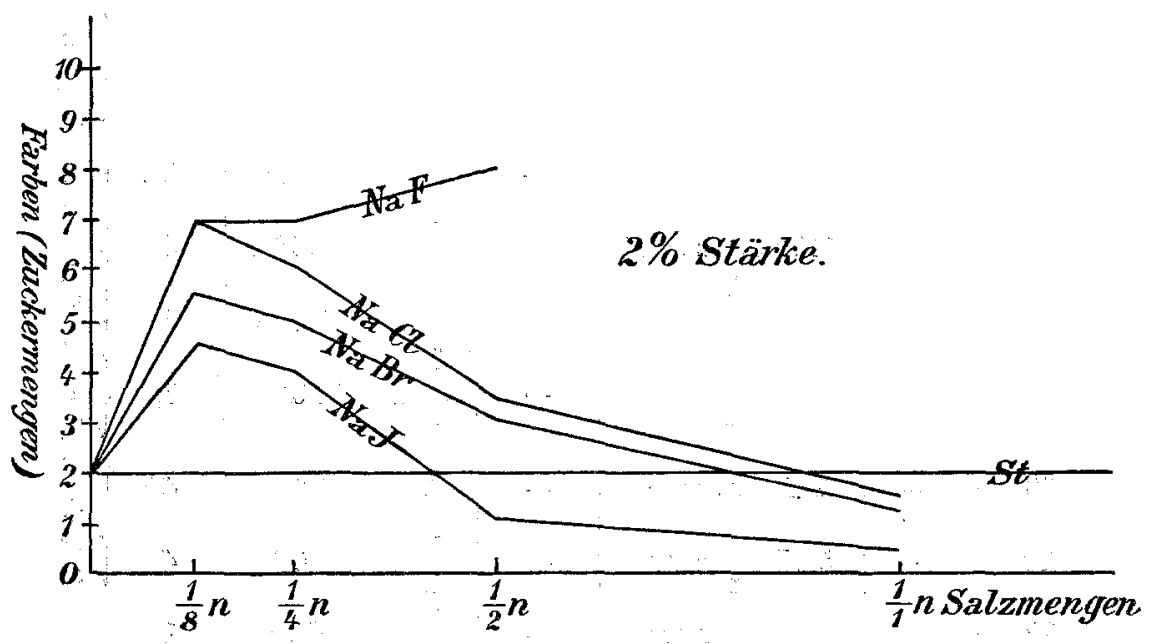

Fig. 4.

der Reihe nach Chlor-, Brom- und Jodnatrium. Dieses letzte hemmt schon verhältnissmässig früh (zwischen $\frac{1}{4} \mathrm{n}$ und $\frac{1}{2} \mathrm{n}$, das ist etwa $5 \%$; Chlor- und Bromnatrium, die in ihren Wirkungen einander ziemlich ähnlich sind, aber erst viel später (zwischen $\frac{1}{2} \mathrm{n}$ und $\frac{1}{1} \mathrm{n}$, das ist etwa 4 , bezw. $8 \%$ ).

Ganz Aehnliches haben übrigens Grützner und Pfleiderer ${ }^{\text {) }}$ für das Pepsin ùnd Lörcher ${ }^{2}$ ) für das Labferment gefunden.

1) Pflüger's Archiv Bd. 66 S. 605.1897.

2) Ebenda Bd. 69 S. 141. 1897. 
Ueber die Einwirkung verschiedener chem. Stoffe auf die Thätigkeit etc. 293

4. Die Wirkung von Chlor-, Brom-und Jodkalium auf die Umwandlung der Stärke durch Speichel.

Hierüber geben folgende Versuche Aufschluss.

Versuch 11.

Mit $\mathrm{KCl}(74,4$ Molekulargewichit) und Stärke von $2 \%$.

\begin{tabular}{|c|c|c|c|c|c|c|c|}
\hline $\begin{array}{l}\text { Nummer } \\
\text { dèr } \\
\text { Gläschen }\end{array}$ & $\begin{array}{l}\text { Stärke- } \\
\text { kleister } \\
\text { von } 4 \% \\
\text { in ccm } \\
\end{array}$ & $\begin{array}{l}\text { Salz- bezw; } \\
\mathrm{H}_{2} \mathrm{O} \text {-Mengen } \\
\text { in . ccm }\end{array}$ & $\begin{array}{l}\text { Speichel- } \\
\text { gemisch } \\
\text { in ccm }\end{array}$ & $\begin{array}{r}\text { Normal- } \\
\text { Salzgel } \\
\text { Misch }\end{array}$ & $\begin{array}{l}\text { Procen- } \\
\text { tual- } \\
\text { alt der } \\
\text { ungen }\end{array}$ & \multicolumn{2}{|c|}{$\begin{array}{l}\text { Erzielter } \\
\text { Farbenton } \\
\text { nach } \\
\text { 10. Minuten }\end{array}$} \\
\hline 1 & 5 & $5 \mathrm{H}_{2} \mathrm{O}$ & 0,3 & 0 & 0 & 2 & \\
\hline 2 & - & $5 \mathrm{KCl} \frac{1}{4}$ normal & - & $\frac{1}{8} n$ & $0,9 \%$ & 8 & \\
\hline 3 & - & $5 \mathrm{KCl} \frac{1}{2}$ normal & - & $\frac{1}{4} \mathrm{n}$ & $1,9 \%$ & 6 & 离 \\
\hline & - & $5 \mathrm{KCl} \frac{1}{1}$ normal & - & $\frac{1}{2} \mathrm{n}$ & $3,7 \%$ & & 峁 \\
\hline 5 & - & $5 \mathrm{KCl} \frac{2}{1}$ normal & - & $\frac{1}{1} \mathrm{n}$ & $7,4 \%$ & & \\
\hline
\end{tabular}

Versuch 12.

Mit KBr (119,0 Molekulargewicht) und Stärke von $2 \%$.

\begin{tabular}{|c|c|c|c|c|c|c|c|}
\hline $\begin{array}{c}\text { Nummer } \\
\text { der } \\
\text { Gläschen }\end{array}$ & $\begin{array}{l}\text { Stärke- } \\
\text { kleister } \\
\text { von } 4 \% \\
\text { in ccm }\end{array}$ & $\begin{array}{c}\text { Salz- bezw. } \\
\mathrm{H}_{2} \mathrm{O} \text {-Mengen } \\
\text { in cem }\end{array}$ & $\begin{array}{l}\text { Speichel- } \\
\text { gemisch } \\
\text { in } \mathrm{ccm}\end{array}$ & $\begin{array}{c}\text { Normal- } \\
\text { Salzge } \\
\text { Miscl }\end{array}$ & $\begin{array}{l}\text { Procen- } \\
\text { tual- } \\
\text { alt der } \\
\text { ungen }\end{array}$ & \multicolumn{2}{|c|}{$\begin{array}{l}\text { Erzielter } \\
\text { Farbenton } \\
\text { nach } \\
\text { 10 Minuten }\end{array}$} \\
\hline 1 & 5 & $5 \mathrm{H}_{2} \mathrm{O}$ & 0,3 & 0 & 0 & 2 & \\
\hline 2 & $\div$ & $5 \mathrm{KBr} \frac{1}{4}$ normal & - & $\frac{1}{8} \mathbf{n}$ & $1,48 \%$ & 6 & \\
\hline & $i$ & $5 \mathrm{KBr} \frac{1}{2}$ normal & - & $\frac{1}{4} \mathrm{n}$ & $2,96 \%$ & 4 & 菢 \\
\hline 4 & $T$ & $5 \mathrm{KBr} \frac{1}{1}$ normal & $\div$ & $\frac{1}{2} n$ & $5,95 \%$ & 4 & 象 \\
\hline 5 & - & $5 \mathrm{KBr} \frac{2}{1}$ normal & $\div$ & $\frac{1}{1} n$ & $11,9 \quad \%$ & 4 & \\
\hline
\end{tabular}


Versuch 13.

Mit KJ (166,0 Molekulargewicht) und Stärke von $2 \%$.

\begin{tabular}{c|c|c|c|c|c|c}
\hline $\begin{array}{c}\text { Nummer } \\
\text { der } \\
\text { Gläschen }\end{array}$ & $\begin{array}{c}\text { Stärke- } \\
\text { Kleister } \\
\text { von } 4 \% \\
\text { in ccm }\end{array}$ & $\begin{array}{c}\text { KJ- bezw. } \\
\mathrm{H}_{2} \mathrm{O} \text {-Mengen } \\
\text { in ccm }\end{array}$ & $\begin{array}{c}\text { Speichel- } \\
\text { gemisch } \\
\text { in ccm }\end{array}$ & $\begin{array}{c}\text { Normal- } \\
\text { Salzgehalt der } \\
\text { Mischungen }\end{array}$ & $\begin{array}{c}\text { Procen- } \\
\text { tual }\end{array}$ & $\begin{array}{c}\text { Erzielter } \\
\text { Farbenton } \\
\text { nach } \\
\text { Minuten }\end{array}$ \\
\hline 1 & 5 & $5 \mathrm{H}_{2} \mathrm{O}$ & 0,3 & 0 & 0 & 2 \\
$\mathbf{2}$ & - & $5 \mathrm{KJ} \frac{1}{4}$ normal & - & $\frac{1}{8} \mathrm{n}$ & $2,6 \%$ & 4 \\
3 & - & $5 \mathrm{KJ} \frac{1}{2}$ normal & - & $\frac{1}{4} \mathrm{n}$ & $4,1 \%$ & 3 \\
4 & - & $5 \mathrm{KJ} \frac{1}{1}$ normal & - & $\frac{1}{2} \mathrm{n}$ & $8,3 \%$ & $3\}$ \\
5 & - & $5 \mathrm{KJ} \frac{2}{1}$ normal & - & $\frac{1}{1} \mathrm{n}$ & $16,6 \%$ & 1
\end{tabular}

Versuch 14.

Mit $\mathrm{KCl}, \mathrm{KBr}$ und $\mathrm{KJ}$ und Stärke von $2 \%$.

\begin{tabular}{|c|c|c|c|c|c|c|c|}
\hline $\begin{array}{c}\text { Nummer } \\
\text { der } \\
\text { Gläschen }\end{array}$ & $\begin{array}{l}\text { Stärke- } \\
\text { kleister } \\
\text { von } 4 \% \\
\text { in ccm }\end{array}$ & $\begin{array}{l}\text { Salz- bezw. } \\
\mathrm{H}_{2} \mathrm{O} \text {-Mengen } \\
\text { in ccm }\end{array}$ & $\begin{array}{l}\text { Speichel- } \\
\text { gemisch } \\
\text { in ccm }\end{array}$ & $\begin{array}{r}\text { Normal- } \\
\text { Salzgeh } \\
\text { Nisch }\end{array}$ & $\begin{array}{l}\text { Procen- } \\
\text { tual } \\
\text { alt der } \\
\text { angen }\end{array}$ & \multicolumn{2}{|c|}{$\begin{array}{c}\text { Erzielter } \\
\text { Farbenton } \\
\text { nach } \\
10 \text { Minuten }\end{array}$} \\
\hline 1 & 5 & $5 \mathrm{H}_{2} \mathrm{O}$ & 0,3 & 0 & 0 & 2 & \\
\hline 2 & - & $5 \mathrm{KCl} \frac{1}{4}$ normal & - & $\frac{1}{8} n$ & $0,9 \%$ & 8 & \\
\hline 3 & - & $5 \mathrm{KCl} \frac{1}{2}$ normal & - & $\frac{1}{4} \mathrm{n}$ & $1,9 \%$ & 7 & E \\
\hline 4 & - & $5 \mathrm{KCl} \frac{1}{1}$ normal & - & $\frac{1}{2} \mathrm{n}$ & $3,7 \%$ & 6 & $\stackrel{5}{\circ}$ \\
\hline 5 & - & $5 \mathrm{KCl} \frac{2}{1}$ normal & - & $\frac{1}{1} \mathrm{n}$ & $7,4 \%$ & 4 & \\
\hline 6 & - & $5 \mathrm{KBr} \frac{1}{4}$ normal & - & $\frac{1}{8} n$ & $1,48 \%$ & 6 & \\
\hline 7 & - & $5 \mathrm{KBr} \frac{1}{2}$ normal & - & $\frac{1}{4} \mathrm{n}$ & $2,96 \%$ & 6 & 这 \\
\hline 8 & - & $5 \mathrm{KBr} \frac{1}{1}$ normal & 一 & $\frac{1}{2} \mathrm{n}$ & $5,95 \%$ & 5 & \\
\hline 9 & - & $5 \mathrm{KBr} \frac{2}{1}$ normal & - & $\frac{1}{1} \mathrm{n}$ & $11,9 \%$ & 4 & \\
\hline 10 & - & $5 \mathrm{KJ} \frac{1}{4}$ normal & - & $\frac{1}{8} \mathfrak{n}$ & $2,6 \%$ & 4 & \\
\hline 11 & - & $5 \mathrm{KJ} \frac{1}{2}$ normal & - & $\frac{1}{4} \mathrm{n}$ & $4,1 \%$ & 3,5 & 0 \\
\hline 12 & - & $5 \mathrm{KJ} \frac{1}{1}$ normal & - & $\frac{1}{2} \mathrm{n}$ & $8,3 \%$ & 3 & \\
\hline 13 & - & $5 \mathrm{KJ} \frac{2}{1}$ normal & - & $\frac{1}{1} \mathrm{n}$ & $16,6 \%$ & 1 & \\
\hline
\end{tabular}


Ueber die Einwirkung verschiedener chem. Stoffe auf die Thätigkeit etc. 295

Wie bei den Halogenverbindungen des Natriums ist auch bei denen des Kaliums die Jodverbindung die am meisten schädigende. Während die $\frac{1}{1}$ normal Lösungen von $\mathrm{KCl}$ und $\mathrm{KBr}$ die Verzuckerung noch befördern, hemmt eine $\frac{1}{1}$ normal Lösung des KJ schon deutlich. Umstehender Versuch 14 auf Seite 294 zeigt die 3 Salze mit einander verglichen und ist in Figur 5 in Curven wiedergegeben.

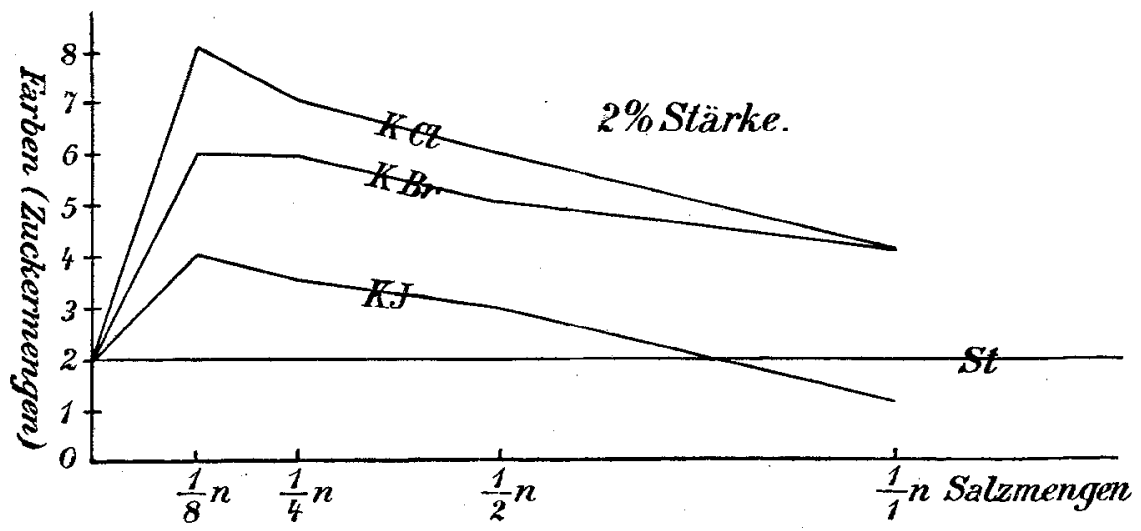

Fig. 5.

Versuch 15.

Mit $\mathrm{NaCl}, \mathrm{NaBr}$ und $\mathrm{NaJ}$ einerseits und $\mathrm{KCl}, \mathrm{KBr}$ und $\mathrm{KJ}$ andererseits und Stärke von $2 \%$.

\begin{tabular}{|c|c|c|c|c|c|c|}
\hline $\begin{array}{l}\text { Nummer } \\
\text { der } \\
\text { Gläschen }\end{array}$ & $\begin{array}{l}\text { Stärke- } \\
\text { kleister } \\
\text { von } 4 \% \% \\
\text { in ccm }\end{array}$ & $\begin{array}{l}\text { Salz- bezw. } \mathrm{H}_{2} \mathrm{O}- \\
\text { Mengen in } \mathrm{ccm}\end{array}$ & $\begin{array}{l}\text { Speichel- } \\
\text { gemisch } \\
\text { in ccm }\end{array}$ & $\begin{array}{r}\text { Normal- } \\
\text { Salzge } \\
\text { Misc }\end{array}$ & $\begin{array}{l}\text { Procen- } \\
\text { tual } \\
\text { alt der } \\
\text { ungen }\end{array}$ & $\begin{array}{c}\text { Erzielter } \\
\text { Farbenton } \\
\text { nach } \\
10 \text { Minuten }\end{array}$ \\
\hline 1 & 5 & $5 \mathrm{H}_{2} \mathrm{O}$ & 0,33 & 0 & 0 & 2 \\
\hline 2 & - & $5 \mathrm{NaCl} \frac{1}{4}$ normal & - & $\frac{1}{2} \mathrm{n}$ & $2,9 \%$ & 51 \\
\hline 3 & - & $5 \mathrm{NaBr} \frac{1}{4}$ normal & - & $\frac{1}{2} \mathrm{n}$ & $5,0 \%$ & $4 \int \frac{\omega}{\omega}$ \\
\hline 4 & - & $5 \mathrm{NaJ} \frac{1}{4}$ normal & - & $\frac{1}{2} \mathrm{n}$ & $7,5 \%$ & $1,5\}$ \\
\hline 5 & - & $5 \mathrm{KCl} \frac{1}{4}$ normal & - & $\frac{1}{2} \mathrm{n}$ & $3,7 \%$ & \\
\hline 6 & - & $5 \mathrm{KBr} \frac{1}{4}$ normal & - & $\frac{1}{2} \mathrm{n}$ & $5,95 \%$ & 总 \\
\hline 7 & - & $5 \mathrm{KJ} \frac{1}{4}$ normal & - & $\frac{1}{2} \mathrm{n}$ & $8,3 \%$ & 3 \\
\hline
\end{tabular}


Die Wirkung der Kalisalze unterscheidet sich von derjenigen der gleichartigen Natronsalze merkwürdiger Weise dadurch, dass sie durchweg die Speichelwirkung besser unterstützen als diese, und dass namentlich die Kalisalzlösungen viel stärker sein dürfen, ohne zu schädigen, als die entsprechenden Natronsalze. So befördert eine KCl-Lösung von $\frac{1}{1}$ normal. Salzgehalt $=7,4 \% \mathrm{KCl}$ die Verzuckerung bedeutend, während eine NaCl-Lösung von $\frac{1}{1}$ normal Salzgehalt $=$ $5,8 \%$ bei einem 2 procentigen Stärkekleister unter sonst gleichen Bedingungen schon deutlich hemmt.

5. Wirkung von Basen auf die Umwandlung von Stärke durch Speichel.

Die, wie schon oben erwähnt, gewöhnliche Angabe, dass die Ptyalinwirkung wesentlich in alkalischer Lösung gut von statten gehe und durch diese unterstützt werde, konnte ich nicht bestätigen. Vielmehr fand ich, dass jede, auch die schwächste alkalische Reaction unter allen Umständen hemmend auf die Verzuckerung der Stärke einwirkte. Meine Versuche stellte ich mit Kali- und Natronlange und mit Ammoniak an. Am stärksten schädigend wirkte die Kalilauge, weniger die Natronlauge und noch weniger das Ammoniak, wie folgende Versuche beweisen.

Versuch 16.

Mit $\mathrm{KOH}(56,0$ Molekulargewicht) und Stärke von $2 \%$.

\begin{tabular}{|c|c|c|c|c|c|c|c|}
\hline $\begin{array}{l}\text { Nr. } \\
\text { der } \\
\text { Gläs- } \\
\text { chen }\end{array}$ & $\begin{array}{l}\text { Stärke- } \\
\text { kleister } \\
\text { von } 4 \% \\
\text { in ccm }\end{array}$ & $\begin{array}{c}\mathrm{KOH} \text { - besw. } \\
\mathrm{H}_{2} \mathrm{O} \text {-Mengen } \\
\text { in ccm }\end{array}$ & $\begin{array}{l}\text { Speichel- } \\
\text { gemisch } \\
\text { in ccm }\end{array}$ & $\begin{array}{l}\text { Normal- } \\
\text { Basenge } \\
\text { Misch }\end{array}$ & $\begin{array}{l}\text { Procen- } \\
\text { tual- } \\
\text { ehalt der } \\
\text { hungen }\end{array}$ & \multicolumn{2}{|c|}{$\begin{array}{c}\text { Erzielter } \\
\text { Farbenton } \\
\text { nach } \\
10 \mathrm{Min} .\end{array}$} \\
\hline 1 & 5 & $5 \mathrm{H}_{2} \mathrm{O}$ & 0,3 & 0 & 0 & 7 & \\
\hline 2 & - & $5 \mathrm{KOH} \frac{1}{10000}$ normal & - & $\frac{1}{20000} \mathrm{n}$ & $0,00028 \%$ & 5 & \\
\hline 3 & - & $5 \mathrm{KOH} \frac{1}{5000}$ normal & - & $\frac{1}{10000} \mathrm{n}$ & $0,0005 \%$ & 2 & \\
\hline 4 & - & $5 \mathrm{KOH} \frac{1}{2500}$ normal & - & $\frac{1}{5000} \mathrm{n}$ & $0,001 \%$ & 2 & \\
\hline 5 & - & $5 \mathrm{KOH} \frac{1}{500}$ normal & - & $\frac{1}{1000} n$ & $0,005 \%$ & $1 / 2$ & \\
\hline 6 & 一 & $5 \mathrm{KOH} \frac{1}{50}$ normal & - & $\frac{1}{100} \mathrm{n}$ & $0,056 \%$ & 0 & \\
\hline
\end{tabular}


Ueber die Einwirkung verschiedener chem. Stoffe auf die Thätigkeit etc. 297

Von etwa $\frac{1}{500} \mathrm{n}$ an war die Reaction der Stärkegemische auf empfindliches Lakmuspapier unwirksam, während die reinen Lösungen bis über $\frac{1}{2000} \mathrm{n}$ auf mein Lakmuspapier alkalisch reagirten.

Weitere Einzelversuche mit $\mathrm{NaOH}$ und $\mathrm{NH}_{3}$ theile ich im Speciellen nicht mit, da sie im Wesentlichen dieselben Ergebnisse wie Versuch 16 lieferten, sondern nur einen Vergleichsversuch zwischen allen drei Basen (s. Versuch 17), der übrigens, so wie alle folgenden Versuche mit einem neuen, stärker wirkenden Speichelgemisch angestellt wurde.

\section{Versuch 17.}

Mit $\mathrm{KOH}, \mathrm{NaOH}, \mathrm{NH}_{3}$ und Stärke von $2 \%$.

\begin{tabular}{|c|c|c|c|c|c|c|c|}
\hline $\begin{array}{l}\text { Nr. } \\
\text { der } \\
\text { Gläs- } \\
\text { chen }\end{array}$ & \begin{tabular}{|l|} 
Stärke- \\
kleister \\
von $4 \%$ \\
in $\mathrm{ccm}$ \\
\end{tabular} & $\begin{array}{l}\text { Basen- bezw. } \\
\mathrm{H}_{2} \mathrm{O} \text {-Mengen } \\
\text { in ccm }\end{array}$ & $\begin{array}{l}\text { Speichel- } \\
\text { gemisch } \\
\text { in ccm }\end{array}$ & $\begin{array}{l}\text { Basengehalt der } \\
\text { Mischungen }\end{array}$ & $\begin{array}{l}\text { Procen- } \\
\text { tual- } \\
\text { ehalt der } \\
\text { ungen }\end{array}$ & \multicolumn{2}{|c|}{$\begin{array}{l}\text { Erzielter } \\
\text { Farbenton } \\
\therefore \text { nach } \\
10 \text { Min. }\end{array}$} \\
\hline 1 & 5 & $5 \mathrm{H}_{2} \mathrm{O}$ & 0,3 & 0 & 0 & 8 & \\
\hline 2 & - & $5 \mathrm{KOH} \frac{1}{5000}$ normal & - & $\frac{1}{10000} \mathrm{n}$ & $0,0005 \%$ & 6 & \\
\hline 3 & - & $5 \mathrm{KOH} \frac{1}{2500}$ normal & - & $\frac{1}{5000} \mathrm{n}$ & $0,001 \%$ & 5 & \\
\hline 4 & - & $5 \mathrm{NaOH} \frac{1}{5000}$ normal & - & $\frac{1}{10000} \mathrm{n}$ & $0,0004 \%$ & 7 & 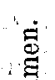 \\
\hline 5 & 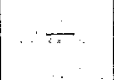 & $.5 \mathrm{NaOH} \frac{1}{2500}$ normal & - & $\frac{1}{5000} \mathrm{n}$ & $0,0008 \%$ & $6^{1 / 2}$ & $\stackrel{g}{ت}$ \\
\hline 6 & - & $5 \mathrm{NH}_{3} \frac{1}{5000}$ normal & $-i$ & $\frac{1}{10000} \mathrm{n}$ & $0,00017 \%$ & $71 / 2$ & \\
\hline 7 & - & $5 \mathrm{NH}_{3} \frac{1}{2500}$ normal & - & $\frac{1}{5000} \mathrm{n}$ & $0,0003 \%$ & $7^{1 / 2}$ & \\
\hline
\end{tabular}

Die Reaction sämmtlicher Glạschen ist gegenüber Lakmus neutral.

Selbst die schwächsten alkalischen Lösungen zeigten sich niemals förderlich. Eine Verdünnung von 1:20000 normal, die auf Lakmuspapier nicht mehr alkalisch reagirte, hemmte, wie in Versuch 16 zu erkennen ist, noch deutlich. Wie alkalische Salze wirken, habe ich nicht näher untersucht, doch habe ich mich von der Richtigkeit der Schierbeck'schen Angaben überzeugt, dass auch sie, wenn auch lange nicht in so hohem Maasse wie die fixen Alkalien, doch unter allen Umständen schädigend wirken. Es muss hiernach anf das Allerentschiedenste mit der alten Angabe gebrochen werden, dass der Mundspeichel am besten in alkalischer Reaction wirke. 
Ich muss mich hier durchweg der Ansicht von Schierbeck anschliessen, welcher, wie schon oben erwähnt wurde, die gleiche Anschauung vertritt und namentlich die fördernde Wirkung der Kohlensäure auf neutralen oder alkalisch reagirenden Speichel nachgewiesen hat. Es muss also in Zukunft der Satz gelten: Das Speichelferment wird durch keinerlei alkalische Reaction unterstützt, sondern im Gegentheil geschädigt.

6. Wirkung der Säuren auf die Umwandlung der Stärke durch Speichel.

Obwohl bereits frühere Angaben dahin gingen, dass schwache Säuren die Ptyalinwirkung nicht hemmten, so ist es doch erst von Schierbeck für Kohlensäure, und von Chittenden, Griswold und Ely ${ }^{1}$ ) für Salzsäure bewiesen worden, dass schwach saure Reaction die Speichelwirkung unterstützt.

Dass diese Angaben an und für sich eine gewisse Wahrscheinlichkeit für sich haben, geht - nebenbei bemerkt - aus folgender Thatsache hervor. Verschiedene Forscher wie $\mathrm{Kjeldah1^{2 }}$ ), Soxhlet ${ }^{3}$ ) und Andere haben unzweifelhaft nachgewiesen, dass pflanzliche diastatische Fermente bei weitem am besten in schwach saurer Reaction ihre Wirkung entfalten. Am deutlichsten tritt diese befördernde Wirkung dann hervor, wenn die angewendete Stärke, was nicht selten vorkommt, schwach alkalisch reagirt. Dann wirkt die Diastase allein fast gar nicht; fügt man ihr aber geringe Säuremengen hinzu, welche eine wenn auch noch so schwach saure Reaction der Gemische zur Folge haben, so beobachtet man eine stark saccharificirende Wirkung.

Dass nun ganz Aehnliches auch von dem Mundspeichel gilt, lehren meine gleich mitzutheilenden Versuche. Ich prüfte Salzsäure, Salpetersäure, Schwefelsäure, Oxalsäure und Essigsäure und stellte die meisten Versuche mit der stärksten und uns am meisten interessirenden Salzsäure an. Sie seien desshalb auch an erster Stelle erwähnt.

1) American. chem. Journal 1881 und 1882, citirt nach Schierbeck, Scandinavisches Archiv für Physiologie Bd. 3 S. 344 (363), 1892, woselbst auch die weitere Literatur zusammengestellt ist.

2) Mittheilungen des Carlsberger Laboratoriums 1879 (schwedisch).

3) Zeitschrift der Vereine für die Rübenzuckerindustrie des deutschen Reiches Bd. 31 S. 656, 1881. (Citirt nach Schierbeck.) 
Ueber die Einwirkung verschiedener chem. Stoffe auf die Thätigkeit etc. 299

\section{Versuch 18.}

Mit $\mathrm{HCl}(36,3$ Molekulargewicht) und Stärke von $4 \%$.

$\left.\begin{array}{c|c|c|c|c|c|c}\hline \hline \begin{array}{c}\text { Nr. } \\ \text { der } \\ \text { Gläs- } \\ \text { chen }\end{array} & \begin{array}{c}\text { Stärke- } \\ \text { kleister } \\ \text { von } 8 \% \\ \text { in ccm }\end{array} & \begin{array}{c}\text { HCl- bezw. } \mathrm{H}_{2} \mathrm{O}- \\ \text { Mengen in ccm }\end{array} & \begin{array}{c}\text { Speichel- } \\ \text { gemisch } \\ \text { in ccm }\end{array} & \begin{array}{c}\text { Normal- } \\ \text { Säuregehalt der } \\ \text { Mischungen }\end{array} & \begin{array}{c}\text { Procen- } \\ \text { tual- }\end{array} & \begin{array}{c}\text { Erzielter } \\ \text { Farbenton } \\ \text { nach } \\ 20 \text { Min. }\end{array} \\ \hline 1 & 5 & 5 \mathrm{H}_{2} \mathrm{O} & 0,3 & 0 & 0 & 2 \\ 2 & - & 5 \mathrm{HCl} \frac{1}{200} \text { normal } & - & \frac{1}{400} \mathrm{n} & 0,009 \% & 12 \\ 3 & - & 5 \mathrm{HCl} \frac{1}{100} \text { normal } & - & \frac{1}{200} \mathrm{n} & 0,018 \% & 10 \\ 4 & - & 5 \mathrm{HCl} \frac{1}{50} \text { normal } & - & \frac{1}{100} \mathrm{n} & 0,036 \% & 1 \\ 5 & - & 5 \mathrm{HCl} \frac{1}{25} \text { normal } & - & \frac{1}{50} \mathrm{n} & 0,0725 \% & 1 / 2 \\ 6 & - & 5 \mathrm{HCl} \frac{1}{12,5} \text { normal } & - & \frac{1}{25} \mathrm{n} & 0,145 \% & 0\end{array}\right)$

Versuch 19.

Mit $\mathrm{HCl}$ und Stärke von $2 \%$.

\begin{tabular}{|c|c|c|c|c|c|c|}
\hline $\begin{array}{l}\text { Nr. } \\
\text { der } \\
\text { Gläs- } \\
\text { chen }\end{array}$ & \begin{tabular}{|c|} 
Stärke- \\
kleister \\
von $4 \%$ \\
in ccm
\end{tabular} & $\begin{array}{l}\mathrm{HCl} \text { - bezw. } \mathrm{H}_{2} \mathrm{O} \text { - } \\
\text { Mengen in } \mathrm{ccm}\end{array}$ & $\mid \begin{array}{c}\text { Speichel- } \\
\text { gemisch } \\
\text { in ccm }\end{array}$ & $\begin{array}{r}\text { Normal- } \\
\text { Säureg } \\
\text { Misc }\end{array}$ & $\begin{array}{l}\text { Procen- } \\
\text { tual- } \\
\text { halt der } \\
\text { ungen }\end{array}$ & $\begin{array}{c}\text { Erzielter } \\
\text { Farbenton } \\
\text { nach } \\
10 \mathrm{Min} .\end{array}$ \\
\hline 1 & 5 & $5 \mathrm{HCl}$ & 0,3 & 0 & 0 & 4 \\
\hline 2 & - & $5 \mathrm{HCl} \frac{1}{1000}$ normal & - & $\frac{1}{2000} n$ & $0,0018 \%$ & 12 \\
\hline 3 & - & $5 \mathrm{HCl} \frac{1}{500}$ normal & - & $\frac{1}{1000} \mathrm{n}$ & $0,003 \%$ & $13\}$ \\
\hline 4 & - & $5 \mathrm{HCl} \frac{1}{200}$ normal & - & $\frac{1}{400} \mathrm{n}$ & $0,009 \%$ & 12 \\
\hline 5 & - & $5 \mathrm{HCl} \frac{1}{100}$ normal & 一 & $\frac{1}{200} \mathbf{n}$ & $0,018 \%$ & $0)$ \\
\hline 6 & - & $5 \mathrm{HCl} \frac{1}{50}$ normal & - & $\frac{1}{100} n$ & $0,03 \%$ & 0 \\
\hline 7 & - & $5 \mathrm{HCl} \frac{1}{25}$ normal & - & $\frac{1}{50} \mathrm{n}$ & $0,07 \%$ & 0 \\
\hline
\end{tabular}




\section{Versuch 20.}

Mit HCl und Stärke von $1 \%$.

\begin{tabular}{c|c|c|c|c|c|c}
\hline \hline $\begin{array}{c}\text { Nr. } \\
\text { der } \\
\text { Gläs- } \\
\text { chen }\end{array}$ & $\begin{array}{c}\text { Stärke- } \\
\text { vleister } \\
\text { von } 2 \% \\
\text { in ccm }\end{array}$ & $\begin{array}{c}\text { HCl- bezw. } \mathrm{H}_{2} \mathrm{O}- \\
\text { Nengen in ccm }\end{array}$ & $\begin{array}{c}\text { Speichel- } \\
\text { gemisch } \\
\text { in ccm }\end{array}$ & $\begin{array}{c}\text { Normal- } \\
\text { Säuregehalt der } \\
\text { Mischungen }\end{array}$ & $\begin{array}{c}\text { Procen- } \\
\text { tual- }\end{array}$ & $\begin{array}{c}\text { Erzielter } \\
\text { Farbenton } \\
\text { nach } \\
10 \text { Min. }\end{array}$ \\
\hline 1 & 5 & $5 \mathrm{H}_{2} \mathrm{O}$ & 0,3 & 0 & 0 & 5 \\
2 & - & $5 \mathrm{HCl} \frac{1}{800}$ normal & - & $\frac{1}{1600} \mathrm{n}$ & $0,002 \%$ & 6 \\
3 & - & $5 \mathrm{HCl} \frac{1}{400}$ normal & - & $\frac{1}{800} \mathrm{n}$ & $0,004 \%$ & 6 \\
4 & - & $5 \mathrm{HCl} \frac{1}{200}$ normal & - & $\frac{1}{400} \mathrm{n}$ & $0,009 \%$ & 0 \\
5 & - & $5 \mathrm{HCl} \frac{1}{100}$ normal & - & $\frac{1}{200} \mathrm{n}$ & $0,018 \%$ & 0
\end{tabular}

Figur 6, 7 und 8 auf Seite 302 stellen die Ergebnisse des Versuches 20 a graphiseh dar. $\mathrm{Zu}$ ihrem Verständniss sei auf die Erklärung der früheren Figuren verwiesen und noch einmal besonders bemerkt, dass der Stärkekleister stets neutral reagirte.

Es ergab sich biernach für diese Sänre, welche als die stärkste aller uns bekannten Säuren und als Bestandtheil des Magensaftes ganz besonderes Interesse hat, dass schwache Lösungen in hohem Maasse die Ptyalinwirkung unterstützen, dass stärkere sie dagegen, wie schon früher bekannt, in gleicher Weise hemmen bezw. ganz aufheben. Ausserdem zeigte sich, wie schon bei den Versuchen mit Kochsalz erwähnt wurde (vergl. Fig. 1,2 und 3 mit 6, 7 und 8), dass die Hemmung um so später, d. h. bei um so stärkerer Säureconcentration, eintrat, je dicker der Stärkekleister war. Es trat bei einer 4 procentigen Stärkekleisterlösung Beförderung bei einem Säuregehalt von $\frac{1}{200}$ normal ein, bei einem 2 procentigen Stärkekleister hemmte $\frac{1}{200}$ normal Säuregehalt schon sehr stark; $\frac{1}{400}$ normal beförderte intensiv. Bei einem 1 procentigen Stärkekleister war dagegen $\frac{1}{400}$ normal Säuregehalt schon hinreichend, um die Umsetzung der Stärke in Zucker hintanzuhalten. Erst bei $\frac{1}{800}$ normal Säuregehalt trat Beförderung der Umsetzung der Stärke in Zucker ein. Die Curvenzüge der Figuren 6,7 und 8 zeigen diese Verhältnisse sehr 
Ueber die Einwirkung verschiedener chem. Stoffe auf die Thätigkeit etc. 301

Versuch 20 a,

Mit HCl und Stärkekleister von $4 \%, 2 \%$ und $1 \%$.

\begin{tabular}{|c|c|c|c|c|c|c|}
\hline $\begin{array}{l}\text { Nummer } \\
\text { der } \\
\text { Gläschen }\end{array}$ & $\begin{array}{l}\text { Stärke- } \\
\text { kleister }\end{array}$ & $\begin{array}{l}\mathrm{HCl} \text { - bezw. } \\
\mathrm{H}_{2} \mathrm{O} \text {-Mengen } \\
\text { in ccm }\end{array}$ & $\begin{array}{l}\text { Speichel- } \\
\text { gemisch } \\
\text { in ccm }\end{array}$ & $\begin{array}{r}\text { Normal- } \\
\text { Säurrege } \\
\text { Misc }\end{array}$ & $\begin{array}{l}\text { Procen- } \\
\text { tual- } \\
\text { halt der } \\
\text { ungen }\end{array}$ & $\begin{array}{c}\text { Erzielter } \\
\text { Farbentor } \\
\text { nach } \\
10 \mathrm{Min} .\end{array}$ \\
\hline 1 & $5 \mathrm{ccm} 8 \%$ & $5 \mathrm{H}_{2} \mathrm{O}$ & 0,3 & 0 & 0 & 2 \\
\hline 2 & $5 \mathrm{ccm} 8 \%$ & $5 \mathrm{HCl} \frac{1}{800} \mathrm{n}$ & - & $\frac{1}{1600} n$ & $0,002 \%$ & $\left.4^{1}\right)$ \\
\hline 3 & $5 \mathrm{ccm} 8 \%$ & $5 \mathrm{HCl} \frac{1}{400} \mathrm{n}$ & - & $\frac{1}{800} n$ & $0,004^{\circ} \%$ & 5 \\
\hline 4 & $5 \mathrm{ccm} 8 \%$ & $5 \mathrm{HCl} \frac{1}{200} \mathrm{n}$ & - & $\frac{1}{400} \mathrm{n}$ & $0,009 \%$ & 6 \\
\hline 5 & $5 \mathrm{ccm} 8 \%$ & $5 \mathrm{HCl} \frac{1}{100} \mathbf{n}$ & - & $\frac{1}{200} \mathbf{n}$ & $0,018 \%$ & 6 \\
\hline 6 & $5 \mathrm{ccm} 8 \%$ & $5 \mathrm{HCl} \frac{1}{50} \mathrm{n}$ & - & $\frac{1}{100} \mathbf{n}$ & $0,036 \%$ & $11 / 2$ \\
\hline 7 & $5 \operatorname{ccm~} 4 \%$ & $5 \mathrm{H}_{2} \mathrm{O}$ & - & 0 & 0 & 4 \\
\hline 8 & $5 \mathrm{ccm} 4 \%$ & $5 \mathrm{HCl} \frac{1}{800} \mathrm{n}$ & 一 & $\frac{1}{1600} \mathrm{n}$ & $0,002 \%$ & 8 \\
\hline 9 & $5 \mathrm{ccm} 4 \%$ & $5 \mathrm{HCl} \frac{1}{400} \mathrm{n}$ & - & $\frac{1}{800} n$ & $0,004 \%$ & 12 \\
\hline 10 & $5 \mathrm{ccm} 4 \%$ & $5 \mathrm{HCl} \frac{1}{200} \mathrm{n}$ & - & $\frac{1}{400} n$ & $0,009 \%$ & 12 \\
\hline 11 & $5 \mathrm{ccm} 4 \%$ & $5 \mathrm{HCl} \frac{1}{100} \mathrm{n}$ & - & $\frac{1}{200} \mathrm{n}$ & $0,018 \%$ & 3 \\
\hline 12 & $5 \mathrm{ccm} 2 \%$ & $5 \mathrm{H}_{2} \mathrm{O}$ & - & 0 & 0 & 6 \\
\hline 13 & $\left.5 \mathrm{ccm} 2^{\circ}\right|_{0}$ & $5 \mathrm{HCl}{ }_{800}^{1} \mathrm{n}$ & - & $\frac{1}{1600} \mathrm{n}$ & $0,002 \%$ & 13 \\
\hline 14 & $5 \mathrm{ccm} 2 \%$ & $5 \mathrm{HCl} \frac{1}{400} \mathrm{n}$ & - & $\frac{1}{800} \mathbf{n}$ & $0,004 \%$ & 14 \\
\hline 15 & $5 \mathrm{ccm} \mathrm{2 \%}$ & $5 \mathrm{HCl} \frac{1}{200} \mathrm{n}$ & - & $\frac{1}{400} \mathbf{n}$ & $0,009 \%$ & $\{$ 丞 \\
\hline
\end{tabular}

schön und übersichtlich und besser als die ganz äbnlichen in den Figuren 1,2 und 3 , weil die letzteren viel kürzer sind und die. Wirkungen sämmtlicher Säuregemische deuttlich und unverkürzt übersehen lassen.

1) $\frac{1}{1600}$ Normal-Lösung reagirte nur noch ganz schwach sauer gegenüber Lakmuspapier, während die Mischungen mit neutral reagirendem Stärkekleister ihre saure Reaction schon bei stärkerem Säuregehalt verloren. 
Ich untersuchte dann weiter, wie oben kurz mitgetheilt, noch verschiedene andere Säuren. Alle wirkten in schwachen Lösungen befördernd, in stärkeren natürlich hemmend. Es würde den Leser

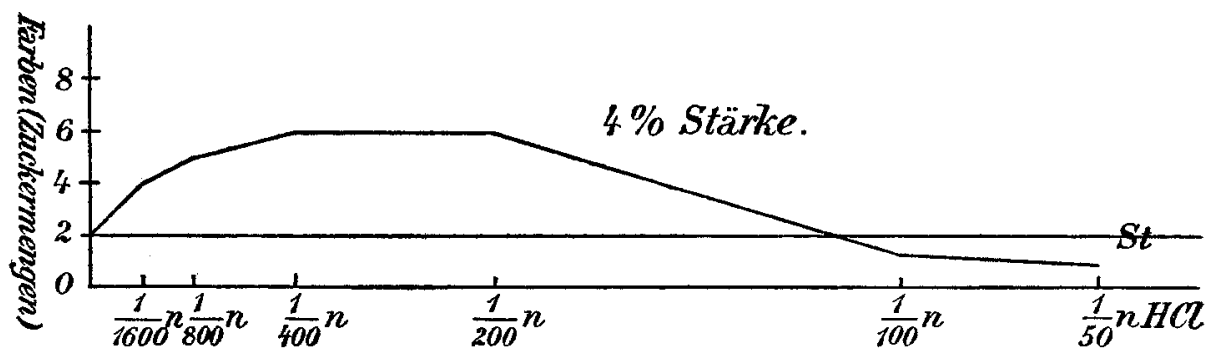

Fig. 6.

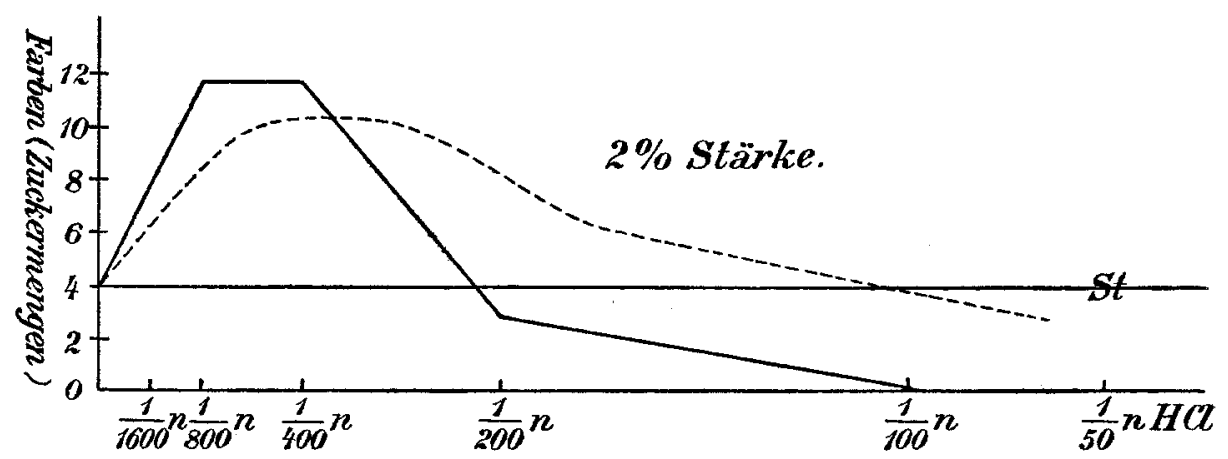

Fig. 7.

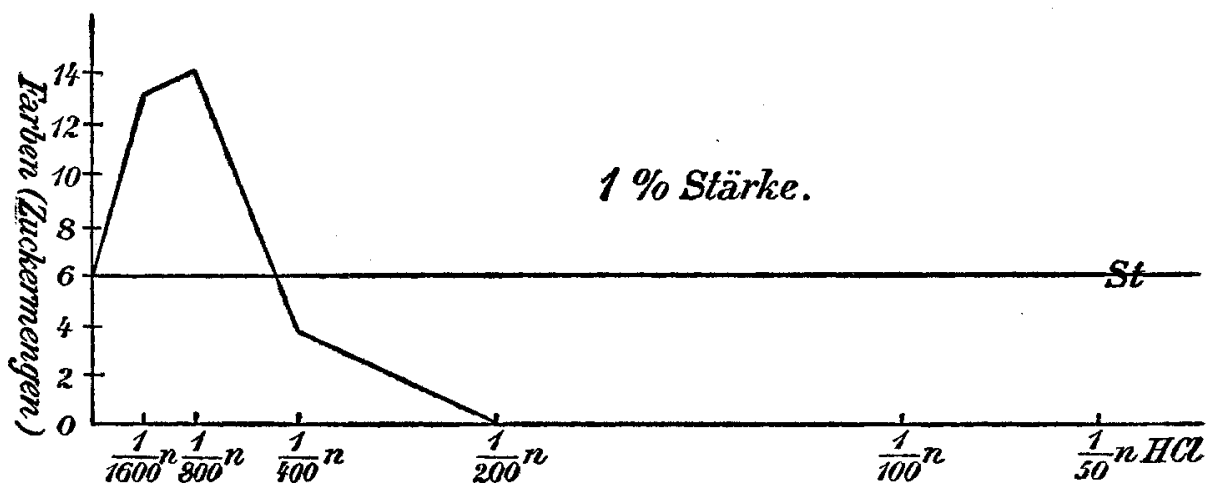

Fig. 8.

ermüden, wenn ich alle meine einzelnen Versuchsreihen ausführlich mittheilen wollte. Es möge also die Bemerkung genügen, dass alle von uns untersuchten Säuren auf Stärkekleister von 
Ueber die Einwirkung verschiedener ehem. Stoffe auf die Thätigkeit etc. 303

$2 \%$ von etwa $\frac{1}{500}$ n abwärts in hohem Gradebefördernd wirkten und je nach der Stärke der Säure verschieden schnell ihre befördernde Wirkung einstellten.

Am lehrreichsten ist ein Vergleich zwischen Salzsäure und Essigsäure, wie er in der Figur 7 graphisch dargestellt ist. Die ausgezogene Curve zeigt die Beförderung der Fermentwirkung durch die Salzsäure, die punktirte, aus verschiedenen Versuchen zusammengesetzt, diejenige der Essigsäure.

Zunächst zeigt sich, dass die Essigsäure in schwachen Lösungen unter der Salzsäure, wenn auch manchmal nur sehr wenig, zurückbleibt, in stärkeren sie dagegen übertrifft. Ferner sieht man, dass verhältnissmässig starke Essigsäuregemische (bis zu $\frac{1}{100} \mathrm{n}$ hin) die Verzuckerung befördern, während eben so starke Salzsäurelösungen sie schon ganz und gar hemmen. Aehnliches gilt von anderen schwachen und starken Säuren.

Es ist lehrreich, hier die Wirkung einer schwachen Säure (Essigsäure) auf dünnen Stärkekleister von $2 \%$ (s. Fig. 7) zu vergleichen mit der einer starken (Salzsäure) auf dicken von $4 \%$ (s. Fig. 6). Die Curvenzüge sind einander ausserordentlich ähnlich. Beide Curven steigen nicht so hoch an und erreichen viel später die horizontale St, d. b. hemmen erst in viel stärkerer Concentration. In dem ersten Fall ist die unterstützende Wirkung der schwachen Säure auf das Ferment an und für sich schwach, in dem zweiten dagegen wird die unterstützende Wirkung der starken Säure dureh die dicke Stärke ${ }^{1}$ ) abgeschwächt.

1) Weitere vergleichende Untersuchungen mit verschieden starken Säuren und verschiedenen Stärkegemischen sind erwünscht. Sie sind augenblicklich im Gange, aber noch nicht abgeschlossen. Im Wesentlichen trifft man auf Verhältnisse wie in Fig. 7 bei Salz- and bei Essigsäure. Wie ferner Säuren im Verein mit Salzen wirken, darüber liegen auch noch keine Untersuchungen vor.

Auch mag hier auf die Aehnlichkeit hingewiesen werden, welche besteht zwischen unseren Versuchen und der Wirkung der Säuren bei der Inversion des Rohrzuckers oder bei der Zersetzung der Ester. Es wird den Säuren hier von den Chemikern (ich halte mich an das treffliche Buch von W. Nernst, Theoret. Chemie S. 420 u. 507, Stuttgart 1898) eine katalytische Kraft zugeschrieben, indem dieselben den Verlauf der betreffenden Reaction, die auch ohne sie stattfinden könnte, stark beschleunigen. Ganz das Gleiche findet bei 
Wenn ich also oben den Satz aussprach, dass jedwede, auch die schwächste alkalische Reaction, die man z. B. vermittelst empfindlichen Lakmuspapiers nicht mehr nachweisen kann, hemmend auf die Speichelthätigkeit einwirkt, so zeigt sich jetzt, dass schwach saure Reaction namentlich von starken Säuren, wie von der Salzsäure, diese Wirkung in allerhöchstem Maasse befördert.

Hiernach liegt es sehr nahe, anzunehmen, dass die Wirkung des Speichels im sauren Magensaft nicht nur nicht gehemmt, sondern bedeutend gefördert wird. Wenn man freilich die Wirkung des Speichels in einem Salzsäuregemisch untersucht, welches in seiner Stärke etwa derjenigen des Magensaftes gleichkommt, so hört unweigerlich die Speichelwirkung auf. Ehe aber diese saure Reaction in dem eben hinabgeschluckten, mit schleimigem Speichel durchsetzten Mageninhalt ganz und gar eindringt, herrschen sicherlich diejenigen Säureconcentrationen in dem Mageninhalt vor, welche die Verzuckerung der Stärke am meisten befördern. Mit doppeltem Recht kann man also jenes erste Stadium der Verdauung (natürlich nur bei denjenigen Geschöpfen, die einen diastatisch wirksamen Mundspeichel haben) das amylolytische nennen, wie dies zweckmässiger Weise von Ellenberger ${ }^{1}$ ) geschehen ist, nicht bloss, weil

den oben erwähnten Versuchen statt, und es sind auch hier höchstwahrscheinlich die freien Wasserstoff-Ionen, welche diese Wirkung ausüben.

Hierfür spricht, dass die Zucker bildende Wirkung des Speichels im Allgemeinen um so mehr befördert wird, je mehr Wasserstoff-Ionen sich in der Lösung befinden. Wenn freilich die Inversionsgeschwindigkeit mit der Concentration der Säuren zunimmt, so gilt das bei uns nur in sehr engen Grenzen und in um so engeren, je stärker die Säuren sind. (Siehe Fig. 7.) Wohl aber wirken unzweifelhaft die starken Säuren (wie die Salzsäure) unter sonst gleichen Bedingungen entschieden energischer als die schwachen Säuren, freilich ebenfalls nur in ziemlich engen Grenzen; denn jede stärkere Säureconcentration schädigt eben das Ferment.

Dass auch Salze allein befördernd auf Inversion des Zuckers einwirken, zeigte meines Wissens zuerst $\mathrm{Nas}$ s. In welcher Weise man deren befördernde Wirkung, allerdings bei Anwesenheit von Säuren, erklärt, darüber hat Arrhenius Anschauungen entwickelt, welche den oben erwähnten über die Wirkung der Wasserstoff-Ionen ähnlich sind.

Warum schliesslich die freien Alkalien die Speichelreaction hindern oder ganz aufheben, wird man einfach dadurch begründen, dass sie eben die offenbar für den Fermentationsvorgang nöthigen, beziehungsweise förderlichen WasserstoffIonen durch ihre freien Hydroxyl-Ionen beseitigen. Grützner.

1) Archiv für wissenschaftliche und praktische Thierheilkunde Bd. 11 s. 249 und Bd. 12 S. 126, 1886 und Pflüger's Archiv Bd. 41 S. 484, 1887. 
Ueber die Einwirkung verschiedener chem. Stoffe auf die Thätigkeit etc. 305 die Verzuckerung des Amylum in dem Magen weiter fortgesetzt wird, sondern weil sie sicherlich durch die Säurewirkung sogar in hohem Maasse unterstützt wird. Dies ist um so sicherer anzunehmen, als nach den interessanten und lehrreichen Untersuchungen von $\mathrm{E}$ wald ${ }^{1}$ ) und Boas sich in den ersten Stunden der Verdauung im menschlichen Magen kaum Salzsäure, sondern nur die viel schwächere Milchsäure nachweisen lässt. Aber auch freie Salzsäure würde entschieden die Verzuckerung der Stärke befördern; denn lange bevor die Säure in den dicken, mit schleimigem und alkalischem Speichel durchsetzten Stärkebrei und in die gleicher Weise durchtränkten dicklichen Brocken eindringt, hat sie - wie unsere Versuche zeigen in wenigen Minuten die Wirkung des Speichels gewiss mehr als verdoppelt.

Zum Schluss möge es mir auch an dieser Stelle gestattet sein, meinem verehrten Lehrer, Herrn Prof. Grützner, der mich bei der Ausführung meiner Arbeit andauernd mit Rath und That unterstützt hat, meinen aufrichtigen Dank zu sagen.

1) Virchow's Archiv Bd. 101 S. 325. 1885. 